\title{
Optimal Control Strategies for the Infectiology of Brucellosis
}

\author{
Nkuba Nyerere $\mathbb{D}^{1,2}{ }^{1,2}$ Livingstone S. Luboobi, ${ }^{3}$ Saul C. Mpeshe $\mathbb{D}^{1},{ }^{4}$ and Gabriel M. Shirima ${ }^{5}$ \\ ${ }^{1}$ Department of Applied Mathematics and Computational Sciences, \\ Nelson Mandela African Institution of Science and Technology, P. O. Box 447, Arusha, Tanzania \\ ${ }^{2}$ Department of Mathematics, Informatics and Computational Sciences, Sokoine University of Agriculture, P. O. Box 3038, \\ Morogoro, Tanzania \\ ${ }^{3}$ Institute of Mathematical Sciences, Strathmore University, P. O. Box 59857-00200, Nairobi, Kenya \\ ${ }^{4}$ Department of Mathematics, University of Iringa, P. O. Box 200, Iringa, Tanzania \\ ${ }^{5}$ Department of Global Health and Bio-Medical Sciences, Nelson Mandela African Institution of Science and Technology, \\ P. O. Box 447, Arusha, Tanzania
}

Correspondence should be addressed to Nkuba Nyerere; emmankuba@sua.ac.tz

Received 28 February 2020; Accepted 22 April 2020; Published 11 May 2020

Academic Editor: Vladimir V. Mityushev

Copyright @ 2020 Nkuba Nyerere et al. This is an open access article distributed under the Creative Commons Attribution License, which permits unrestricted use, distribution, and reproduction in any medium, provided the original work is properly cited.

Brucellosis is a zoonotic infection caused by Gram-negative bacteria of genus Brucella. The disease is of public health, veterinary, and economic significance in most of the developed and developing countries. Direct contact between susceptible and infective animals or their contaminated products are the two major routes of the disease transmission. In this paper, we investigate the impacts of controls of livestock vaccination, gradual culling through slaughter of seropositive cattle and small ruminants, environmental hygiene and sanitation, and personal protection in humans on the transmission dynamics of Brucellosis. The necessary conditions for an optimal control problem are rigorously analyzed using Pontryagin's maximum principle. The main ambition is to minimize the spread of brucellosis disease in the community as well as the costs of control strategies. Findings showed that the effective use of livestock vaccination, gradual culling through slaughter of seropositive cattle and small ruminants, environmental hygiene and sanitation, and personal protection in humans have a significant impact in minimizing the disease spread in livestock and human populations. Moreover, cost-effectiveness analysis of the controls showed that the combination of livestock vaccination, gradual culling through slaughter, environmental sanitation, and personal protection in humans has high impact and lower cost of prevention.

\section{Introduction}

Brucellosis is a zoonotic infection caused by Gram-negative bacteria of genus Brucella which includes; B. abortus primarly from cattle, $B$. melitensis from small ruminants, $B$. suis from swine, and B. canis from dogs [1-4]. It is considered by the Food and Agriculture Organisation (FAO), the World Health Organisation (WHO), and World Organization for Animal Health (Office International des Epizooties (OIE)) as one of the most widespread zoonoses in the world alongside bovine tuberculosis and rabies [5]. The disease is an ancient one that was described more than 2000 years ago by the Romans [6] and has been known by various names, including Mediterranean fever, Malta fever, gastric remittent fever, Bang's disease, Crimean fever, Gibraltar fever, rock fever, lazybones disease, and undulant fever [7].

Brucella bacteria was first isolated in 1887 from an infected individual's blood by a British military medical officer David Bruce and by that reason the disease was named brucellosis to honor his contribution [8]. Furthermore, in 1905, Zamitt carried out an experiment on goats to investigate the origin of human brucellosis and found that human brucellosis originates from goats [9]. To date, ten brucella species have been identified and primarily named after the features of infection or the animal source. Of these, the following four have moderate-to-significant human pathogenicity: Brucella melitensis (highest pathogenicity), Brucella suis (high pathogenicity), Brucella 
abortus (moderate pathogenicity), and Brucella canis (moderate pathogenicity) [10-12].

Brucellosis is endemic in most of the developing world. It causes devastating losses to the livestock industry especially small-scale livestock holders, thereby limiting economic growth and hindering access to international markets [13]. The economic importance of the disease is based on the fact that it causes financial losses from abortions, sterility, decreased milk production, veterinary fees, and costs of replacement of animals. In animals, brucellosis is transmitted when a susceptible animal ingests contaminated materials such as tissues or discharges from infected animals, while in humans the bacteria is transmitted by ingestion of contaminated unpasteurized milk or other dairy products and direct contact through occupational activities such as farmers, laboratory personnel, abattoir workers, and veterinarians. According to Ducrotoy et al. [14], there are epidemiological situations in which infections of small ruminants by $B$. abortus occur in areas where they are in contact with cattle and B. melitensis is absent. Ducrotoy et al. [14] suggests that coinfections by two different brucellae are rather unlikely because of the development of immunity in an ongoing infection and, in fact, they have never been convincingly proven.

Infected animals exhibit clinical signs that are of economic significance to stakeholders. These signs include reduced fertility, abortion, poor weight gain, lost draught power, and a substantial decline in milk production $[13,15]$. Symptoms in human includes continuous or intermittent fever, headache, weakness, profuse sweats, chills, joint pains, aches, and weight loss, as well as devastating complications that leads to miscarriage in pregnant women. Neurological complications, endocarditis, and testicular or bone abscess formation can also occur $[16,17]$. The infection can also affect the liver and spleen and may last for longer terms if not treated. Furthermore, the clinical signs of brucellosis in humans present diagnostic difficulties because they overlap with those of typhoid fever, malaria, rheumatic fever, joint diseases, and relapsing fever. Human brucellosis is debilitating and requires prolonged treatment with combination of antibiotics [18].

The global burden of human brucellosis remains enormous: The infection causes more than 500,000 new cases per year worldwide. The annual number of reported cases in the United States has dropped significantly to about 100 cases per year due to aggressive animal vaccination programs and milk pasteurization. Most United States cases are now due to the consumption of illegally imported unpasteurized dairy products from Mexico, and approximately $60 \%$ of human brucellosis cases occur in California and Texas [19]. In Africa, brucellosis exists throughout sub-Saharan Africa, but the prevalence is unclear and poorly understood with varying reports from country to country and geographical regions, as well as animal factors [20]. Most African countries have poor socioeconomic status, with people living with and by their livestock, while health networks, surveillance, and vaccination programs are virtually nonexistent. In Tanzania, the first outbreak of brucellosis was reported in Arusha in 1927 [21]. Previous surveys in
Tanzania have demonstrated the occurrence of the disease in cattle in various production systems, regions, and zones with individual animal level seroprevalence varying from 1 to $30 \%$. In humans, the average prevalence varies from 1 to $5 \%$ [22]; a recent study by [23] shows that brucellosis incidence is moderate in northern Tanzania and suggests that the disease is endemic and an important human health problem in this area. Moreover, human cases had been reported in areas of northern, eastern, lake, and western zones with seroprevalence varying from 0.7 to $20.5 \%$ [24, 25]. Despite the WHO, FAO, and OIE efforts and interventions being available, brucellosis continues to pose great economic threat by affecting livelihood and food security in both developed and developing countries from generation to generation. Thus, there is a need to assess the current control strategies and their cost effectiveness if we are to control or eradicate the disease. So far, few studies [10, 26-33] have been developed to analyze dynamics and spread of brucellosis in homogeneous/heterogeneous populations. However, none of these studies had considered the mathematical approach for optimal control and cost effectiveness in reducing or eradicating the disease in cattle, small ruminants, and human populations. In this paper, the dynamics and cost effectiveness of the control strategies for brucellosis using mathematical models are rigorously studied.

\section{Model Formulation}

A mathematical model for the transmission dynamics of brucellosis incorporating the time-dependent controls to some parameters is formulated in this section. Some assumptions used in this section are similar to those in $[27,28]$, but the time-dependent parameters $u_{1}(t), u_{2}(t), u_{3}(t)$, and $u_{4}(t)$ make the difference between our previous brucellosis works and the current work. The most important reason for taking preventive and control measures on brucellosis is to minimize the prevalence of the disease, and if possible eradicate it from the population. Particularly, the level of susceptibility of healthy individuals against the infection is minimized by protective measures, whereas the number of infective individuals in the community is reduced by control measures. In this paper, a time-dependent variable $u_{1}(t)$ is introduced as a control that aims at reducing the number of susceptible animals in the herds and consequently to reduce the disease transmission to the vaccination coverage failure rate $\left(1-u_{1}(t)\right) \lambda_{1}$. In other words, $u_{1}(t)$ is the measure of the effectiveness of S19 and Rev1 vaccine for cattle and small ruminants, and $\left(1-u_{1}(t)\right) \lambda_{1}$ is the failure rate of vaccination for ruminants. That is, if vaccination of ruminant coverage is $100 \%$ effective, zero brucellosis incidence may be recorded in that particular region. Thus, $u_{1}(t)$ aims at reducing the susceptibility level of healthy animals on the disease as well as the disease transmission rate. Based on the fact that there is neither disease-induced deaths nor treatment for infected ruminants, we introduce $u_{2}(t)$, a control variable that measures the efficiency of gradual culling of seropositive animal parameters $d_{c}$ and $d_{s}$ for the infected cattle and small ruminants, respectively. Furthermore, 
gradual culling of seropositive animals targets at curtailing the number of infectious ruminants in the community and consequently reduces the disease transmission rate to $u_{2}(t) d_{c}$ and $u_{2}(t) d_{s}$ in cattle and small ruminants, respectively. To reduce or eliminate the number of Brucella in the environment, the control variable $u_{3}(t)$ is introduced as the measure of effectiveness of environmental hygiene and sanitation parameter $\tau$. In particular, environmental hygiene and sanitation refers to proper disposal of placentas and aborted foetuses. A time-dependent control variable $u_{4}(t)$ is introduced in the model as the measure of the effectiveness of personal protection in humans so that $\left(1-u_{4}(t)\right) \lambda_{1}$ is the failure rate of the control strategy. In this context, personal protection refers to personal hygiene, protection of the environment, food hygiene (adequate boiling of fresh milk intended for drinking or making other milk products), and adoption to safe working practices including use of personal protection equipments such as gloves, masks, eye wear, and closed footwear when handling potentially infected materials such as aborted foetus, placenta, and gravid uterus practices. Based on the fact that treatment of human brucellosis reduces the risk of disease and that has a very low or negligible transmission blocking effect, the time-dependent control for this parameter is not of much concern. To determine the necessary conditions for optimal impact of incorporated parameters, we use Pontryagin's Maximum Principle as the method for obtaining the optimal combination of incorporated controls. The aim is to limit and prevent the spread of brucellosis disease and at the same time the cost of administering these controls is minimized.

2.1. Model Assumptions. Formulation of the optimal control model is guided by the following assumptions:

(i) The mixing of individuals in each population is homogeneous

(ii) There is no direct transmission between cattle and small ruminants

(iii) Infected animals shed Brucella pathogens in the environment

(iv) Livestock seropositivity is life-long lasting

(v) Immunized individuals cannot be infected unless they are resistant to infection wanes

(vi) There is a constant natural mortality rate in each of the species

(vii) The birth rate for each population is greater than the natural mortality rate

The compartmental diagram with the time-dependent control strategies is shown in Figure 1, whereas the variables and parameters used in this model are, respectively, summarized in Tables 1 and 2.

2.2. Compartmental Flow Diagram for the Disease Dynamics. The interactions between human, cattle, small ruminant populations, and Brucella in the environment are illustrated in Figure 1.

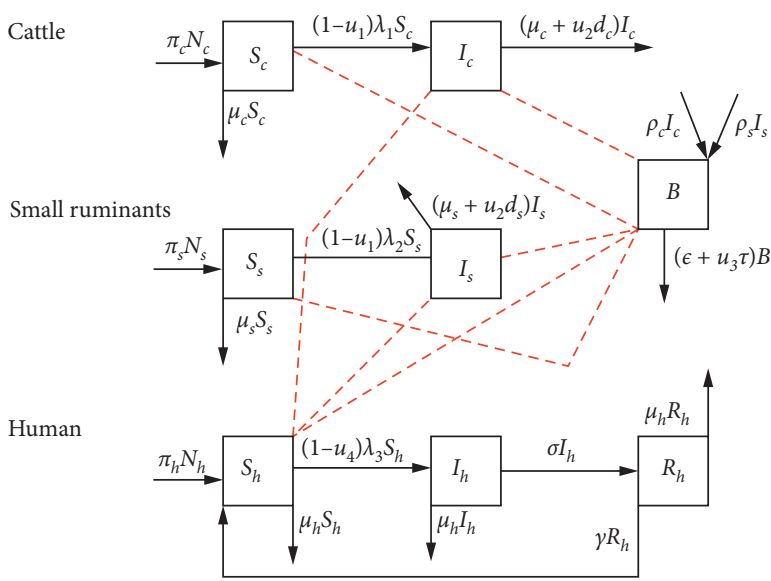

FIgURE 1: A schematic diagram for direct and indirect transmission of brucellosis in cattle, small ruminant, and human populations. Solid arrows represent transfer of individuals from one subpopulation to another while dotted lines represent interactions that lead to infection.

The resulting model is shown as a system of differential equations:

$$
\begin{aligned}
\frac{\mathrm{d} S_{c}}{\mathrm{~d} t}= & \pi_{c} N_{c}-\left(\left(1-u_{1}(t)\right)\left(\beta_{c} I_{c}+\alpha_{c} B\right)+\mu_{c}\right) S_{c}, \\
\frac{\mathrm{d} I_{c}}{\mathrm{~d} t}= & \left(1-u_{1}(t)\right)\left(\beta_{c} I_{c}+\alpha_{c} B\right) S_{c}-\left(\mu_{c}+u_{2}(t) d_{c}\right) I_{c}, \\
\frac{\mathrm{d} S_{s}}{\mathrm{~d} t}= & \pi_{s} N_{s}-\left(\left(1-u_{1}(t)\right)\left(\beta_{s} I_{s}+\alpha_{s} B\right)+\mu_{s}\right) S_{s}, \\
\frac{\mathrm{d} I_{s}}{\mathrm{~d} t}= & \left(1-u_{1}(t)\right)\left(\beta_{s} I_{s}+\alpha_{s} B\right) S_{s}-\left(\mu_{s}+u_{2}(t) d_{s}\right) I_{s}, \\
\frac{\mathrm{d} S_{h}}{\mathrm{~d} t}= & \pi_{h} N_{h}+\gamma R_{h}-\left(( 1 - u _ { 4 } ( t ) ) \left(\beta_{h c}(t) I_{c}+\beta_{h s} I_{s}\right.\right. \\
& \left.\left.+\beta_{h h}(t) I_{h}+\alpha_{h} B\right)+\mu_{h}\right) S_{h}, \\
\frac{\mathrm{d} I_{h}}{\mathrm{~d} t}= & \left(1-u_{4}(t)\right)\left(\beta_{h c} I_{c}+\beta_{h s} I_{s}+\beta_{h h} I_{h}+\alpha_{h} B\right) S_{h} \\
& -\left(\sigma+\mu_{h}+d_{h}\right) I_{h}, \\
\frac{\mathrm{d} R_{h}}{\mathrm{~d} t}= & \sigma I_{h}-\left(\gamma+\mu_{h}\right) R_{h}, \\
\frac{\mathrm{d} B}{\mathrm{~d} t}= & \rho_{c} I_{c}+\rho_{s} I_{s}-\left(\varepsilon+u_{3}(t) \tau\right) B .
\end{aligned}
$$

\section{Model Properties}

3.1. Invariant Region. In this section, we investigate whether model variables have biological interpretation and have a unique bounded solution that exists for all the time. That is solutions of model system (1) with nonnegative initial data remain nonnegative for all time $t \geq 0$. We apply the approach 
TABLe 1: Model variables.

\begin{tabular}{lc}
\hline Variable & Description \\
\hline$S_{h}(t)$ & Number of susceptible humans at time $t$ \\
$I_{h}(t)$ & Number of infected human at time $t$ \\
$R_{h}(t)$ & Number of recovered humans at time $t$ \\
$S_{c}(t)$ & Number of susceptible cattle at time $t$ \\
$I_{c}(t)$ & Number of infected cattle at time $t$ \\
$S_{s}(t)$ & Number of susceptible small ruminants at time $t$ \\
$I_{s}(t)$ & Number of infected small ruminants at time $t$ \\
$B(t)$ & Number of Brucella bacteria load per unit volume in the environment at time $t$ \\
\hline
\end{tabular}

TABLE 2: Model parameters used in the model and their description.

\begin{tabular}{lc}
\hline Parameter & Description \\
\hline$\pi_{c}$ & Per capita cattle birth rate \\
$\pi_{h}$ & Per capita human birth rate \\
$\sigma$ & Human recovery rate \\
$\mu_{h}$ & Per capita human natural death rate \\
$\beta_{c}$ & Within cattle transmission rate \\
$\alpha_{c}$ & Brucella from the environment to cattle transmission rate \\
$\alpha_{s}$ & Brucella from the environment to small ruminant transmission rate \\
$\alpha_{h}$ & Brucella from the environment to human transmission rate \\
$\beta_{c h}$ & Cattle to human transmission rate \\
$\beta_{s h}$ & Small ruminants to human transmission rate \\
$\pi_{s}$ & Small ruminants per capita birth rate \\
$\beta_{s}$ & Within small ruminant transmission rate \\
$\mu_{s}$ & Per capita small ruminant natural death rate \\
$\tau$ & Environmental hygiene and sanitation rate \\
$\varepsilon$ & Decaying rate of Brucella in the environment \\
$d_{c}$ & Culling rate of seropositive cattle \\
$d_{s}$ & Culling rate of seropositive small ruminants \\
\hline
\end{tabular}

in $[27,28]$ to the optimal control model (1). Model system (1) where can be expressed in the compact form as follows:

$$
\frac{\mathrm{d} X}{\mathrm{~d} t}=M X+F
$$

$$
M=\left[\begin{array}{cccccccc}
-d_{0} & 0 & 0 & 0 & 0 & 0 & 0 & 0 \\
\left(1-u_{1}\right) \lambda_{1} & -d_{3} & 0 & 0 & 0 & 0 & 0 & 0 \\
0 & 0 & -d_{1} & 0 & 0 & 0 & 0 & 0 \\
0 & 0 & \left(1-u_{1}\right) \lambda_{2} & -d_{4} & 0 & 0 & 0 & 0 \\
0 & 0 & 0 & 0 & -d_{2} & -\left(\mu_{s}+u_{2} d_{s}\right) & 0 & 0 \\
0 & 0 & 0 & 0 & \left(1-u_{4}\right) \lambda_{3} & -\left(\sigma+\mu_{h}+d_{h}\right) & 0 & 0 \\
0 & 0 & 0 & 0 & 0 & \sigma & -\left(\gamma+\mu_{h}\right) & 0 \\
0 & \rho_{c} & 0 & \rho_{s} & 0 & 0 & 0 & -\left(\varepsilon+u_{3} \tau\right)
\end{array}\right],
$$


with

$$
\begin{aligned}
d_{0} & =\left(\left(1-u_{1}\right) \lambda_{1}+\mu_{c}\right), \\
d_{1} & =\left(\left(1-u_{1}\right) \lambda_{1}+\mu_{s}\right), \\
d_{2} & =\left(\left(1-u_{4}\right) \lambda_{3}+\mu_{h}\right), \\
d_{3} & =\left(\mu_{c}+u_{2} d_{c}\right), \\
d_{4} & =\left(\mu_{s}+u_{2} d_{s}\right), \\
X & =\left(S_{c}, I_{c}, S_{s}, I_{s}, S_{h}, I_{h}, R_{h}, B\right),
\end{aligned}
$$

and $F$ is a column vector given by

$$
F=\left(\pi_{c} N_{c}^{0}, 0, \pi_{s} N_{s}^{0}, 0, \pi_{h} N_{h}^{0}, 0,0,0\right)^{T} .
$$

It can be noticed that $M X$ is Meltzer matrix since all of its off-diagonal entries are nonnegative, for all $X \in \mathbb{R}_{+}^{8}$. Therefore, using the fact that $F>0$, the model system (1) is positively invariant in $\mathbb{R}_{+}^{8}$, which means that an arbitrary trajectory of the system starting in $\mathbb{R}_{+}^{8}$ remains in $\mathbb{R}_{+}^{8}$ forever. In addition, the right hand $F$ is Lipschitz continuous. Thus, a unique maximal solution exists and so

$$
\Omega=\left\{\left(S_{c}, I_{c}, S_{s}, I_{s}, S_{h}, I_{h}, R_{h}, B\right) \geq 0\right\} \in \mathbb{R}_{+}^{8},
$$

is the feasible region for model (1). Thus, model (1) is epidemiologically and mathematically well posed in the region $\Omega$.

\section{Model Analysis}

4.1. Disease Free Equilibrium. The Brucellosis free equilibrium point $E^{0}$ for the constant controls case was computed by setting the right-hand side of equations in model system (1) to zero, and it was found to be

$$
E^{0}=\left(S_{c}^{0}, 0, S_{s}^{0}, 0, S_{h}^{0}, 0,0,0\right),
$$

where

$$
\begin{aligned}
& S_{c}^{0}=\frac{\pi_{c} N_{c}^{0}}{\mu_{c}}, \\
& S_{s}^{0}=\frac{\pi_{s} N_{s}^{0}}{\mu_{s}}, \\
& S_{h}^{0}=\frac{\pi_{h} N_{h}^{0}}{\mu_{h}} .
\end{aligned}
$$

4.2. The Effective Reproduction Number. Computation of the effective reproduction number $R_{e}$ for model system (1) using the standard method of the next generation matrix developed by Diekmann et al. $[34,35]$ is carried out in this section. $R_{e}$ is defined as the measure of average number of infections caused by a single infectious individual introduced in a community in which intervention strategies are administered [36]. When there are no interventions or controls, the number of secondary infections caused by typical infected individual during his entire period of infectiousness is called basic reproduction number, $R_{0}$. Upon computation, the effective reproduction number was found to be

$$
\begin{aligned}
R_{e}= & \max \left\{\frac{R_{11}+R_{22}+\sqrt{\left(R_{22}-R_{11}\right)^{2}+4 R_{12} R_{21}}}{2},\right. \\
& \left.\frac{\left(1-u_{4}\right) \beta_{h h} \pi_{h} N_{h}^{0}}{\mu_{h}\left(\sigma+\mu_{h}+d_{h}\right)}\right\},
\end{aligned}
$$

where

$$
\begin{aligned}
& R_{11}=\frac{\left(\left(1-u_{1}\right)\left(\varepsilon+u_{3} \tau\right) \beta_{c}+\alpha_{c} \rho_{c}\right) \pi_{c} N_{c}^{0}}{\mu_{c}\left(\mu_{c}+u_{2} d_{c}\right)\left(\varepsilon+u_{3} \tau\right)}, \\
& R_{12}=\frac{\alpha_{c} \rho_{s} \pi_{c} N_{c}^{0}}{\mu_{c}\left(\mu_{s}+u_{2} d_{s}\right)\left(\varepsilon+u_{3} \tau\right)}, \\
& R_{22}=\frac{\left(\left(1-u_{1}\right)\left(\varepsilon+u_{2} \tau\right) \beta_{s}+\alpha_{s} \rho_{s}\right) \pi_{s} N_{s}^{0}}{\mu_{s}\left(\mu_{s}+u_{2} d_{s}\right)\left(\varepsilon+u_{3} \tau\right)}, \\
& R_{21}=\frac{\alpha_{s} \rho_{c} \pi_{s} N_{s}^{0}}{\mu_{s}\left(\mu_{c}+u_{2} d_{c}\right)\left(\varepsilon+u_{3} \tau\right)} .
\end{aligned}
$$

The first and the second expressions of equation (9) represent, respectively, the effective reproduction numbers in the livestock and human populations. When there are no controls $\left(u_{1}=u_{2}=u_{3}=0\right)$ for the disease in both human population and livestock, the effective reproduction is reduced to the basic reproduction number given by

$$
R_{0}=\max \left\{\frac{R_{11}^{0}+R_{22}^{0}+\sqrt{\left(R_{22}^{0}-R_{11}^{0}\right)^{2}+4 R_{12}^{0} R_{21}^{0}}}{2}, \frac{\beta_{h h} \pi_{h} N_{h}^{0}}{\mu_{h}\left(\mu_{h}+d_{h}\right)}\right\},
$$

where

$$
\begin{aligned}
& R_{11}^{0}=\frac{\left(\varepsilon \beta_{c}+\alpha_{c} \rho_{c}\right) \pi_{c} N_{c}^{0}}{\mu_{c}^{2} \varepsilon}, \\
& R_{12}^{0}=\frac{\alpha_{c} \rho_{s} \pi_{c} N_{c}^{0}}{\mu_{c} \mu_{s} \varepsilon}, \\
& R_{22}^{0}=\frac{\left(\varepsilon \beta_{s}+\alpha_{s} \rho_{s}\right) \pi_{s} N_{s}^{0}}{\mu_{s}^{2} \varepsilon}, \\
& R_{21}^{0}=\frac{\alpha_{s} \rho_{c} \pi_{s} N_{s}^{0}}{\mu_{s} \mu_{c} \varepsilon},
\end{aligned}
$$

as in $[27,28]$. Based on the fact that human-to-human transmission is less than within livestock transmission [37-42] and that environmental contamination by humans is negligible, the effective reproduction number and basic reproductive number are, respectively, given by

$$
R_{e}=\frac{R_{11}+R_{22}+\sqrt{\left(R_{22}-R_{11}\right)^{2}+4 R_{12} R_{21}}}{2}
$$

and 


$$
R_{0}=\frac{R_{11}^{0}+R_{22}^{0}+\sqrt{\left(R_{22}^{0}-R_{11}^{0}\right)^{2}+4 R_{12}^{0} R_{21}^{0}}}{2} .
$$

The numerical simulations for the comparison between effective or control reproductive number and basic reproductive number with respect to variations in some parameters are illustrated in Figures 2 and 3. Parameter values used for the simulations are presented in Table 3.

Figure 2 shows that both $R_{0}$ and $R_{e}$ increases with the increase in effective contact rate between livestock (direct transmission). For instance, when within cattle effective contact rate $\beta_{c}$ is 0.3 , the control reproduction number and basic reproduction are, respectively, 0.3 and 1.4 . On the contrary, if the within small ruminants effective contact rate $\beta_{s}$ is $0.3, R_{0}$ and $R_{e}$ are 1.2 and 0.25 , respectively.

Similarly, Figure 3 shows that both $R_{0}$ and $R_{e}$ increases with the increase in consumption rate of Brucella from the contaminated environment by livestock (indirect transmission). In particular, when Brucella consumption rate by cattle $\alpha_{c}$ is $0.3, R_{0}$ and $R_{e}$ are, respectively, 0.1 and 1.1. On the contrary, if the small ruminants consumption rate of Brucella from the environment $\alpha_{s}$ is $0.3, R_{0}$ and $R_{e}$ are 2 and 1.1, respectively. More importantly, Figure 3(b) reveals that $\alpha_{s}>0.3$ leads to $R_{e}>1$ (disease persistence) and that small ruminants are more susceptible to the contaminated environment than cattle. The possible reasons include small ruminant density and herd turnover due to births and introduction of new animals, as pointed in [43].

Generally, the controls $u_{1}, u_{2}$, and $u_{3}$ have high impact on $R_{e}$ by keeping it always less than $R_{0}$.

4.3. Local Stability of the Equilibria. In this section, the tracedeterminant method is employed to investigate the local stability of the Brucellosis free equilibrium point for the model system (1).

Theorem 1. The disease-free equilibrium for the Brucellosis model system (1) is locally asymptotically stable if $R_{0}<1$ and unstable if $R_{0}>1$.

Proof. We show that the variational matrix $J\left(E_{0}\right)$ of the brucellosis free model system has a negative trace and positive determinant. The Jacobian matrix for system (1) is given by

$$
J\left(E_{0}\right)=\left[\begin{array}{cccccccc}
-\left(1-u_{1}\right) \mu_{c} & -\left(1-u_{1}\right) \beta_{c} S_{c}^{0} & 0 & 0 & 0 & 0 & 0 & -a \\
0 & -a_{1} & 0 & 0 & 0 & 0 & 0 & a \\
0 & 0 & -a_{2} & -a_{3} & 0 & 0 & 0 & -b \\
0 & 0 & 0 & b_{1} & 0 & 0 & 0 & b \\
0 & -\left(1-u_{4}\right) \beta_{h c} S_{h}^{0} & 0 & -b_{2} & -b_{3} & -b_{4} & \gamma & -c \\
0 & \left(1-u_{4}\right) \beta_{h c} S_{h}^{0} & 0 & b_{2} & 0 & c_{1} & 0 & c \\
0 & 0 & 0 & 0 & 0 & \sigma & -\left(\gamma+\mu_{h}\right) & 0 \\
0 & \rho_{c} & 0 & \rho_{s} & 0 & 0 & 0 & -r
\end{array}\right],
$$

where

$$
\begin{aligned}
a & =\left(1-u_{1}\right) \alpha_{c} S_{c}^{0}, \\
a_{1} & =\left(1-u_{1}\right) \beta_{c} S_{c}^{0}-\left(\mu_{c}+u_{2} d_{c}\right) \\
a_{2} & =\left(1-u_{1}\right) \mu_{s} \\
a_{3} & =\left(1-u_{1}\right) \beta_{s} S_{s}^{0} \\
b & =\left(1-u_{1}\right) \alpha_{s} S_{s}^{0} \\
b_{1} & =\left(1-u_{1}\right) \beta_{s} S_{s}^{0}-\left(\mu_{s}+u_{2} d_{s}\right) \\
b_{2} & =\left(1-u_{4}\right) \beta_{h s} S_{h}^{0} \\
b_{3} & =\left(1-u_{4}\right) \mu_{h}, \\
b_{4} & =\left(1-u_{4}\right) \beta_{h h} S_{h}^{0}, \\
c_{1} & =\beta_{h h} S_{h}^{0}-\left(\sigma+\mu_{h}+d_{h}\right), \\
r & =\left(\varepsilon+u_{3} \tau\right) .
\end{aligned}
$$

Direct computation of the Jacobian matrix $J\left(E_{0}\right)$ gives

$$
\begin{aligned}
\operatorname{Tr}\left(J\left(E_{0}\right)\right)= & -\left(\mu_{c}+u_{2} d_{c}\right)\left(1-\frac{\left(1-u_{1}\right) \beta_{c} S_{c}^{0}}{\mu_{c}+u_{2} d_{c}}\right) \\
& -\left(\mu_{s}+u_{2} d_{s}\right)\left(1-\frac{\left(1-u_{1}\right) \beta_{s} S_{s}^{0}}{\mu_{s}+u_{2} d_{s}}\right) \\
& -\left(\sigma+\mu_{h}+d_{h}\right)\left(1-\frac{\left(1-u_{4}\right) \beta_{h h} S_{h}^{0}}{\sigma+\mu_{h}+d_{h}}\right) \\
& \left.-\left(\left(1-u_{1}\right)\left(\mu_{c}+\mu_{s}\right)+\left(1-u_{4}\right) \mu_{h}\right)\right) \\
& -\left(\gamma+\mu_{h}+\varepsilon+u_{3} \tau\right) .
\end{aligned}
$$

Thus, the trace of the Jacobian matrix is less than zero, that is, $\operatorname{Tr}\left(J\left(E_{0}\right)\right)<0$ if 


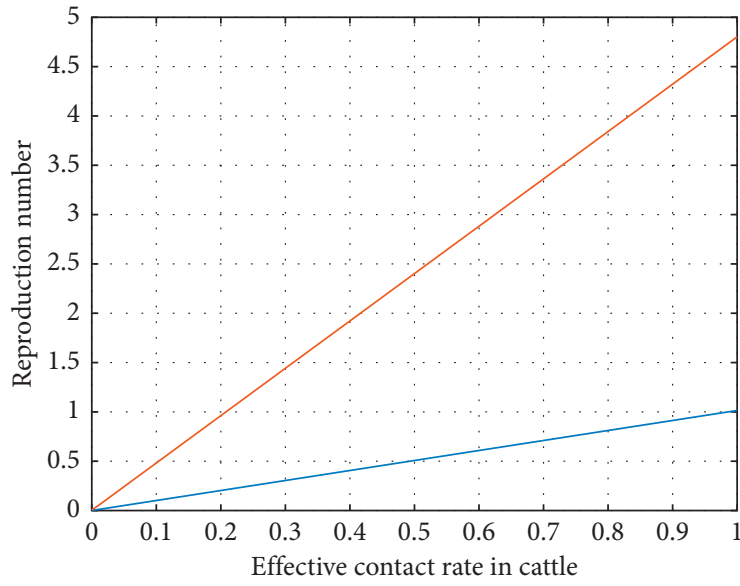

_ Effective reproductive number

_ Basic reproductive number

(a)

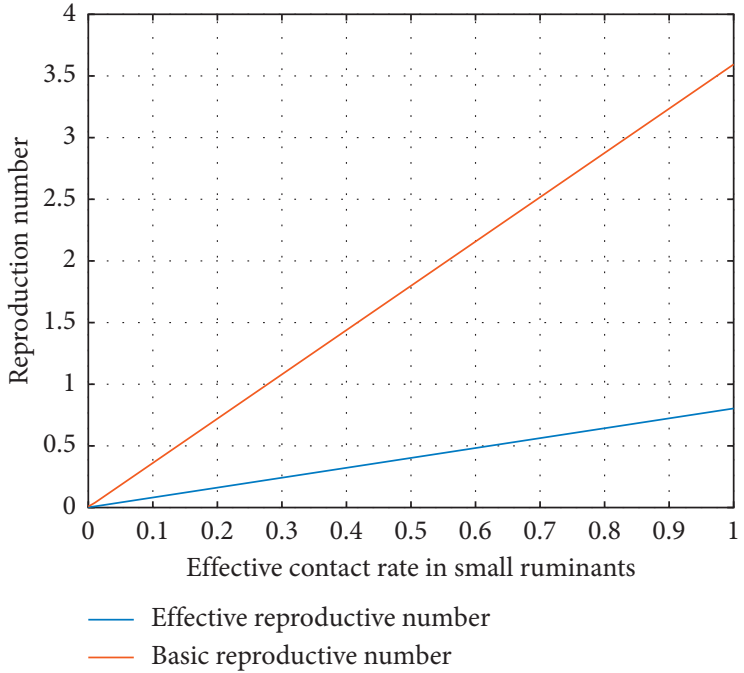

(b)

FiguRE 2: Variations in the reproduction number with respect to changes in effective contact rate in livestock.

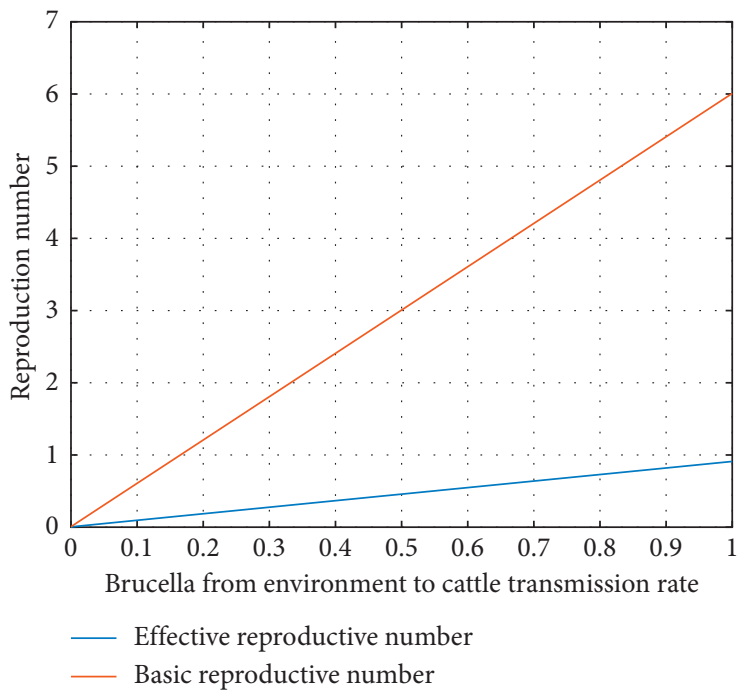

(a)

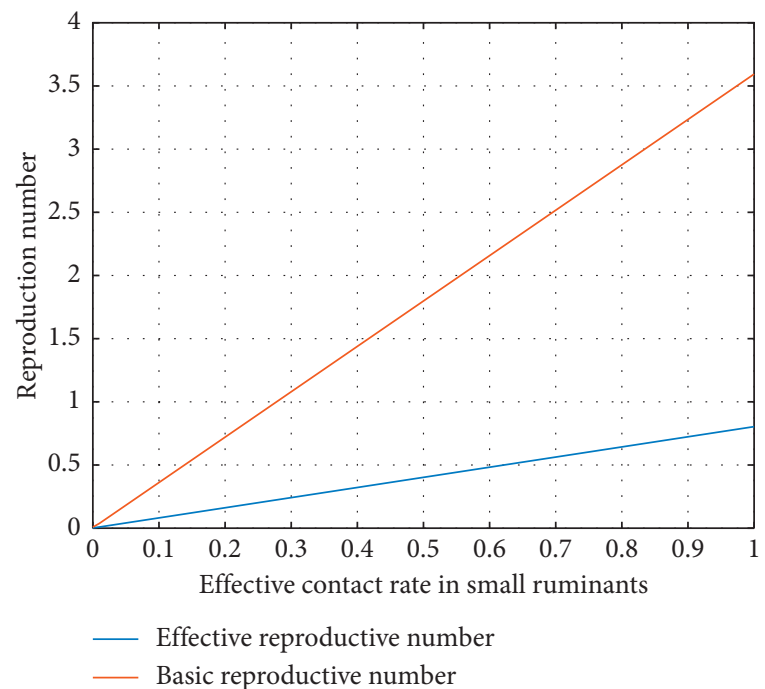

(b)

Figure 3: Variations in the reproduction number with respect to changes of the consumption rate of Brucella from the environment by livestock.

$$
\begin{aligned}
& \frac{\left(1-u_{1}\right) \beta_{c} S_{c}^{0}}{\mu_{c}+u_{2} d_{c}}<1, \\
& \frac{\left(1-u_{1}\right) \beta_{s} S_{s}^{0}}{\mu_{c}+u_{2} d_{s}}<1, \\
& \frac{\left(1-u_{4}\right) \beta_{h h} S_{h}^{0}}{\sigma+\mu_{h}+d_{h}}<1 .
\end{aligned}
$$

On the contrary, the determinant of matrix $J\left(E_{0}\right)$ is computed using Maple 16 Software and was found to be

$$
\begin{aligned}
\operatorname{Det}\left(J\left(E_{0}\right)\right)= & Q_{0}\left(1-R_{h}\right)\left(Q_{1}\left(1-R_{c}\right)+\left(\mu_{s}+u_{2} d_{s}\right)\right. \\
& \left.\cdot\left(1-R_{s}\right)\left(Q_{2}+Q_{3}\left(1-R_{c}\right)\right)\right),
\end{aligned}
$$

where 
TABLE 3: Model parameter values.

\begin{tabular}{lcc}
\hline Parameter & Value & Unit \\
\hline$\pi_{c}$ & 0.3 & year $^{-1}$ \\
$\beta_{c}$ & 0.0011 & year $^{-1}$ \\
$\mu_{c}$ & 0.25 & year $^{-1}$ \\
$d_{c}$ & 0.35 & year $^{-1}$ \\
$\alpha_{c}$ & 0.00035 & year $^{-1}$ \\
$\rho_{c}$ & 10 & year $^{-1}$ \\
$\epsilon$ & 8 & year $^{-1}$ \\
$\tau$ & 12 & year $^{-1}$ \\
$\pi_{s}$ & 0.4 & year $^{-1}$ \\
$\beta_{s}$ & 0.001 & year $^{-1}$ \\
$\mu_{s}$ & 0.35 & year $^{-1}$ \\
$d_{s}$ & 0.4 & year $^{-1}$ \\
$\alpha_{s}$ & 0.00032 & year $^{-1}$ \\
$\rho_{s}$ & 15 & year $^{-1}$ \\
\hline
\end{tabular}

$$
\begin{aligned}
& R_{h}=\frac{\left(1-u_{4}\right) \beta_{h h} S_{h}^{0}}{\sigma+\mu_{h}+d_{h}}, \\
& R_{s}=\frac{\left(1-u_{1}\right) \beta_{s} S_{s}^{0}}{\mu_{s}+u_{2} d_{s}}, \\
& R_{c}=\frac{\left(1-u_{1}\right) \beta_{c} S_{c}^{0}}{\mu_{c}+u_{2} d_{c}}, \\
& Q_{0}=\left(1-u_{1}\right)^{2}\left(1-u_{4}\right)\left(\gamma+\mu_{h}\right)\left(\sigma+\mu_{h}+d_{h}\right) \mu_{c} \mu_{s} \mu_{h}, \\
& Q_{1}=\left(1-u_{1}\right)\left(\mu_{c}+u_{2} d_{c}\right) \alpha_{s} \rho_{s} S_{s}^{0}, \\
& Q_{2}=\left(1-u_{1}\right) \alpha_{c} \rho_{c} S_{c}^{0}, \\
& Q_{3}=\left(\varepsilon+u_{3} \tau\right)\left(\mu_{c}+u_{2} d_{c}\right) .
\end{aligned}
$$

It follows that $\operatorname{Det}\left(J\left(E_{0}\right)\right)>0$ if $R_{h}<1, R_{c}<1$, and $R_{s}<1$. Thus, the Brucellosis free equilibrium for model system (1) is locally asymptotically stable if $R_{h}<1, R_{c}<1$, and $R_{s}<1$. Local stability of the Brucellosis free equilibrium suggests local stability of the endemic equilibrium for the reverse condition [28].

\section{Optimal Control}

To investigate the optimal level of efforts that would be required to control brucellosis infections, we first formulate the objective function $J$ to be minimized subject to the number of ruminants and human infections and cost of applying the controls:

$$
\begin{aligned}
J= & \int_{0}^{t_{f}}\left(A_{1} I_{c}+A_{2} I_{s}+A_{3} B+A_{4} I_{h}+\frac{B_{1} u_{1}^{2}}{2}+\frac{B_{2} u_{2}^{2}}{2}\right. \\
& \left.+\frac{B_{3} u_{3}^{2}}{2}+\frac{B_{4} u_{4}^{2}}{2}\right) \mathrm{d} t,
\end{aligned}
$$

where $A_{1}, A_{2}, A_{3}$, and $A_{4}$ are positive weight constants of infected cattle, infected small ruminants, Brucella in the environment, and infected human classes, respectively. Furthermore, the constants $B_{1}, B_{2}, B_{3}$, and $B_{4}$ are positive weights which balance the cost factors associated with control strategies $u_{1}, u_{2}, u_{3}$, and $u_{4}$, respectively. More importantly, the cost of each control strategy is assumed to be nonlinear and takes the quadratic form that is $B_{1} u_{1}^{2} / 2$ is the cost of control strategy associated with vaccination of ruminants, $B_{2} u_{2}^{2} / 2$ is the cost associated with gradual culling of seropositive animals strategy, $B_{3} u_{3}^{2} / 2$ is the cost associated with environmental hygiene and sanitation, and $B_{4} u_{4}^{2} / 2$ is the cost associated with personal protections in humans.

With the objective function $J\left(u_{1}, u_{2}, u_{3}, u_{4}\right)$, our goal is to minimize the number of infected ruminants and humans, while minimizing the cost of controls, $u_{1}(t), u_{2}(t), u_{3}(t)$, and $u_{4}(t)$. We seek an optimal control $u_{1}^{*}(t), u_{2}^{*}(t), u_{3}^{*}(t)$, and $u_{4}^{*}(t)$ such that

$$
J\left(u_{1}^{*}, u_{2}^{*}, u_{3}^{*}, u_{4}^{*}\right)=\min \left\{J\left(u_{1}, u_{2}, u_{3}, u_{4}\right) \mid u_{1}, u_{2}, u_{3}, u_{4} \in u\right\} \text {, }
$$

where $u=\left\{u_{1}, u_{2}, u_{3}, u_{4}\right\}$ such that $u_{1}, u_{2}, u_{3}$, and $u_{4}$ are measurable with $0 \leq u_{1} \leq 1, \quad 0 \leq u_{2} \leq 1, \quad 0 \leq u_{3} \leq 1$, and $0 \leq u_{4} \leq 1$, for $t \in\left[0, t_{f}\right]$ is the control set.

\section{Existence of an Optimal Control}

Theorem 2. There exists an optimal control set $\left(u_{1}^{*}, u_{2}^{*}, u_{3}^{*}, u_{4}^{*}\right) \in u$ with corresponding nonnegative states $\left(S_{c}, I_{c}, S_{s}, I_{s}, S_{h}, I_{h}, R_{h}\right)$ that minimize the objective functional $J\left(u_{1}(t), u_{2}(t), u_{3}(t), u_{4}(t)\right)$.

Proof. The positivity and uniform boundedness of the state variables as well as the controls on $\left[0, t_{f}\right]$ entail the existence of a minimizing sequence:

$$
J\left(u_{1}^{n}(t), u_{2}^{n}(t), u_{3}^{n}(t), u_{4}^{n}(t)\right)
$$

such that

$$
\begin{aligned}
& \lim _{n \longrightarrow \infty} J\left(u_{1}^{n}(t), u_{2}^{n}(t), u_{3}^{n}(t), u_{4}^{n}(t)\right) \\
& \quad=\inf _{\left(u_{1}^{n}(t), u_{2}^{n}(t), u_{3}^{n}(t), u_{4}^{n}(t)\right) \in u} J\left(u_{1}^{n}(t), u_{2}^{n}(t), u_{3}^{n}(t), u_{4}^{n}(t)\right) .
\end{aligned}
$$

The boundedness of all the state and control variables implies that all the derivatives of the state variables are also bounded. If the corresponding sequence of state variables is denoted by $\left(S_{c}, I_{c}, S_{s}, I_{s}, S_{h}, I_{h}, R_{h}, B\right)$, then all state variables are Lipschitz continuous with the same Lipschitz constant. This implies that the sequence $\left(S_{c}, I_{c}, S_{s}, I_{s}, S_{h}, I_{h}, R_{h}\right)$ is uniformly equicontinuous in $\left[0, t_{f}\right]$. Following the approach in [44], the state sequence has a subsequence that converges uniformly to $\left(S_{c}, I_{c}, S_{s}, I_{s}, S_{h}, I_{h}, R_{h}, B\right)$ in $\left[0, t_{f}\right]$. In addition, we can establish that the control sequence $u^{n}=\left(S_{c}^{n}, I_{c}^{n}, S_{s}^{n}, I_{s}^{n}, S_{h}^{n}, I_{h}^{n}, R_{h}^{n}, B^{n}\right)$ has a subsequence that converges weakly in $L^{2}\left(0, t_{f}\right)$. Let $\left(u_{1}^{*}, u_{2}^{*}, u_{3}^{*}, u_{4}^{*}\right) \in u$ be such that $u_{i}^{n} \longrightarrow u_{i}^{*}$ weakly in $L^{2}\left(0, t_{f}\right)$ for $i=1,2,3,4$. Applying the lower semicontinuity of norms in weak $L^{2}$, we have 


$$
\left\|u_{i}^{*}\right\|_{L^{2}}^{2} \leq \lim _{n \longrightarrow \infty} \inf \left\|u_{i}^{n}(t)\right\|_{L^{2}}^{2}, \quad i=1,2,3,4 .
$$

This means that

$$
\begin{aligned}
J\left(u_{1}^{*}, u_{2}^{*}, u_{3}^{*}, u_{4}^{*}\right) \leq & \lim _{n \longrightarrow \infty} \int_{0}^{t_{f}}\left(A_{1} I_{c}^{n}+A_{2} I_{s}^{2}+A_{3} I_{h}^{n}+A_{4} B_{4}^{n}\right. \\
& \left.+\frac{B_{1} u_{1}^{n}}{2}+\frac{B_{2} u_{2}^{n}}{2}+\frac{B_{3} u_{3}^{n}}{2}+\frac{B_{4} u_{4}^{n}}{2}\right) \mathrm{d} t .
\end{aligned}
$$

Thus, there exists a set of controls $\left(u_{1}^{*}, u_{2}^{*}, u_{3}^{*}, u_{4}^{*}\right)$ that minimizes our objective functional $J\left(u_{1}, u_{2}, u_{3}, u_{4}\right)$.

\section{Characterization of Optimal Control}

In this section, we derive necessary conditions for an optimal control and formulate an optimality system that characterizes the optimal control using upper and lower bound technique. The necessary condition is that an optimal control problem must satisfy Pontryagin's maximum principle [45]. The principle converts system (1) and equation (21) into a problem of minimizing pointwise a Hamiltonian $H$, with respect to $u_{1}, u_{2}, u_{3}$, and $u_{4}$ defined by

$$
\begin{aligned}
H= & A_{1} I_{c}+A_{2} I_{s}+A_{3} B+A_{4} I_{h}+\frac{B_{1} u_{1}^{2}}{2}+\frac{B_{2} u_{2}^{2}}{2}+\frac{B_{3} u_{3}^{2}}{2}+\frac{B_{4} u_{4}^{2}}{2} \\
& +\lambda_{S_{c}}\left(\pi_{c} N_{c}-\left(\left(1-u_{1}\right)\left(\beta_{c} I_{c}+\alpha_{c} B\right)+\mu_{c}\right) S_{c}\right) \\
& +\lambda_{I_{c}}\left(\left(1-u_{1}\right)\left(\beta_{c} I_{c}+\alpha_{c} B\right) S_{c}-\left(\mu_{c}+u_{2}\right) I_{c}\right) \\
& +\lambda_{S_{s}}\left(\pi_{s} N_{s}-\left(\left(1-u_{1}\right)\left(\beta_{s} I_{s}+\alpha_{s} B\right)+\mu_{s}\right) S_{s}\right) \\
& +\lambda_{I_{s}}\left(\left(1-u_{1}\right)\left(\beta_{s} I_{s}+\alpha_{s} B\right) S_{s}-\left(\mu_{s}+u_{2}\right) I_{s}\right) \\
& +\lambda_{B}\left(\rho_{c} I_{c}+\rho_{s} I_{s}-\left(\varepsilon+u_{3} \tau\right) B\right) \\
& +\lambda_{S_{h}}\left(\pi_{h} N_{h}+\gamma R_{h}-\left(\left(1-u_{4}\right)\right.\right. \\
& \left.\left.+\left(\beta_{h c} I_{c}+\beta_{h s} I_{s}+\beta_{h h} I_{h}+\alpha_{h} B\right)+\mu_{h}\right) S_{h}\right) \\
& +\lambda_{I_{h}}\left(\left(1-u_{4}\right)\left(\beta_{h c} I_{c}+\beta_{h s} I_{s}+\beta_{h h} I_{h}+\alpha_{h} B\right) S_{h}\right. \\
& \left.-\left(\sigma+\mu_{h}+d_{h}\right) I_{h}\right) \\
& +\lambda_{R_{h}}\left(\sigma I_{h}-\left(\gamma+\mu_{h}+d_{h}\right) R_{h}\right),
\end{aligned}
$$

where $\lambda_{S_{c}}, \lambda_{I_{c}}, \lambda_{S_{s}}, \lambda_{I_{s}}, \lambda_{B}, \lambda_{S_{h}}, \lambda_{I_{h}}$, and $\lambda_{R_{h}}$ are the adjoint or costate variables.
Applying Pontryagin's maximum principle [45] and the existence result for the optimal control [46], we obtain the following.

Theorem 3. For optimal tetra controls $u_{1}^{*}, u_{2}^{*}, u_{3}^{*}$, and $u_{4}^{*}$ that minimizes $J\left(u_{1}, u_{2}, u_{3}, u_{4}\right)$ over $u$, there exist adjoint variables $\lambda_{S_{c}}, \lambda_{I_{c}}, \lambda_{S_{s}}, \lambda_{I_{s}}, \lambda_{B}, \lambda_{S_{h}}, \lambda_{I_{h}}$, and $\lambda_{R_{h}}$, satisfying

$$
\begin{aligned}
\frac{\mathrm{d} \lambda_{S_{c}}}{\mathrm{~d} t}= & \left(1-u_{1}\right)\left(\beta_{c} I_{c}+\alpha_{c} B\right)\left(\lambda_{S_{c}}-\lambda_{I_{c}}\right)+\mu_{c} \lambda_{S_{c}}, \\
\frac{\mathrm{d} \lambda_{I_{c}}}{\mathrm{~d} t}= & -A_{1}+\left(1-u_{1}\right)\left(\lambda_{S_{c}}-\lambda_{I_{c}}\right) \beta_{c} S_{c}+\lambda_{I_{c}}\left(\mu_{c}+u_{2} d_{c}\right) \\
& +\left(1-u_{4}\right)\left(\lambda_{S_{h}}-\lambda_{I_{h}}\right) \beta_{h c} S_{h}-\rho_{c} \lambda_{B}, \\
\frac{\mathrm{d} \lambda_{S_{s}}}{\mathrm{~d} t}= & \left(1-u_{1}\right)\left(\beta_{s} I_{s}+\alpha_{s} B\right)\left(\lambda_{S_{s}}-\lambda_{I_{s}}\right)+\mu_{s} \lambda_{S_{s}}, \\
\frac{\mathrm{d} \lambda_{I_{s}}}{\mathrm{~d} t}= & -A_{2}+\left(1-u_{1}\right)\left(\lambda_{S_{s}}-\lambda_{I_{s}}\right) \beta_{s} S_{s}+\lambda_{I_{s}}\left(\mu_{s}+u_{2} d_{s}\right) \\
& +\left(1-u_{4}\right)\left(\lambda_{S_{h}}-\lambda_{I_{h}}\right) \beta_{h s} S_{h}-\lambda_{B} \rho_{s}, \\
\frac{\mathrm{d} \lambda_{S_{h}}}{\mathrm{~d} t}= & \left(1-u_{4}\right)\left(\beta_{h c} I_{c}+\beta_{h s} I_{s}+\beta_{h h} I_{h}+\alpha_{h} B\right)\left(\lambda_{S_{h}}-\lambda_{I_{h}}\right) \\
& +\mu_{h} \lambda_{S_{h}}, \\
\frac{\mathrm{d} \lambda_{I_{h}}=}{\mathrm{d} t} & -A_{4}+\left(1-u_{4}\right)\left(\lambda_{S_{h}}-\lambda_{I_{h}}\right) \beta_{h h} S_{h}+\lambda_{I_{h}}\left(\sigma+\mu_{h}+d_{h}\right) \\
& -\sigma \lambda_{R_{h}}, \\
\frac{\mathrm{d} \lambda_{R_{h}}}{\mathrm{~d} t}= & \gamma\left(\lambda_{R_{h}}-\lambda_{S_{h}}\right)+\mu_{h} \lambda_{R_{h}}, \\
\mathrm{~d} t & -A_{3}+\left(1-u_{1}\right)\left(\lambda_{S_{c}}-\lambda_{I_{c}}\right) \alpha_{c} S_{c} \\
& +\left(1-u_{1}\right)\left(\lambda_{S_{s}}-\lambda_{I_{s}}\right) \alpha_{s} S_{s} \\
& +\left(1-u_{4}\right)\left(\lambda_{S_{h}}-\lambda_{I_{h}}\right) \alpha_{h} S_{h}+\lambda_{B}\left(\varepsilon+u_{3} \tau\right), \\
&
\end{aligned}
$$

with transversality conditions:

$$
\begin{aligned}
\lambda_{S_{c}}\left(t_{f}\right) & =\lambda_{I_{c}}\left(t_{f}\right)=\lambda_{S_{s}}\left(t_{f}\right)=\lambda_{I_{s}}\left(t_{f}\right)=\lambda_{B}\left(t_{f}\right)=\lambda_{S_{h}}\left(t_{f}\right) \\
& =\lambda_{I_{h}}\left(t_{f}\right)=\lambda_{R_{h}}\left(t_{f}\right)=0 .
\end{aligned}
$$


The following characterization holds on the interior of the control set $u$ :

$$
\begin{aligned}
& u_{1}^{*}=\max \left\{0, \min \left(1, \frac{1}{B_{1}}\left(\left(\beta_{c} I_{c}+\alpha_{c} B\right)\left(\lambda_{I_{c}}-\lambda_{S_{c}}\right) S_{c}+\left(\beta_{s} I_{s}+\alpha_{s} B\right)\left(\lambda_{I_{s}}-\lambda_{S_{s}}\right) S_{s}\right)\right)\right\} \\
& u_{2}^{*}=\max \left\{0, \min \left(1, \frac{1}{B_{2}}\left(d_{c} I_{c} \lambda_{I_{c}}+d_{s} I_{s} \lambda_{I_{s}}\right) S_{c}\right)\right\} \\
& u_{3}^{*}=\max \left\{0, \min \left(1, \frac{1}{B_{3}} \lambda_{B} B\right)\right\} \\
& u_{4}^{*}=\max \left\{0, \min \left(1, \frac{1}{B_{4}}\left(\beta_{h c} I_{c}+\beta_{h s} I_{s}+\beta_{h h} I_{h}+\alpha_{h} B\right)\left(\lambda_{I_{h}}-\lambda_{S_{h}}\right) S_{h}\right)\right\}
\end{aligned}
$$

where $\lambda_{S_{c}}, \lambda_{I_{c}}, \lambda_{S_{s}}, \lambda_{I_{s}}, \lambda_{B}, \lambda_{S_{h}}, \lambda_{I_{h}}$, and $\lambda_{R_{h}}$ are solutions of equation (29).

Proof. The form of adjoint (or costate) system (28) and transversality conditions (29) are standard results from Pontryagin's Maximum Principle [45]. To obtain the costate system (28), the partial derivatives of the Hamiltonian $(\mathrm{H})$ equation (27) with respect to each state variable are computed as follows:

$$
\begin{gathered}
\frac{\mathrm{d} \lambda_{S_{c}}}{\mathrm{~d} t}=-\frac{\partial H}{\partial S_{c}}, \quad \lambda_{S_{c}}\left(t_{f}\right)=0, \\
\vdots \\
\frac{\mathrm{d} \lambda_{R_{h}}}{\mathrm{~d} t}=-\frac{\partial H}{\partial R_{h}}, \quad \lambda_{R_{h}}\left(t_{f}\right)=0 .
\end{gathered}
$$

The optimality equation (30) is obtained by finding the partial derivative of the Hamiltonian equation (27) with respect to each control variable and solving for the optimal values of $u_{i}^{*}$ where the derivative vanishes. That is, $\left(\partial H / \partial u_{i}\right)=0$ for $i=1,2,3,4$.

Solving for $u_{i}^{*}$ subject to the constraints gives the characterization equation (30). Next, we discuss the numerical solutions of the optimality system and the corresponding results of varying the optimal controls $u_{1}, u_{2}, u_{3}$, and $u_{4}$, the parameter choices, and the interpretations from various cases.

\section{Numerical Simulations}

In this section, we analyze numerically the optimal control strategies for the brucellosis transmission in model system (27). The controls of interest are vaccination of susceptible ruminants, gradual culling of seropositive ruminants, environmental hygiene and sanitation, and personal protection in humans. The optimal control solution is obtained by solving the optimality system which consists of the state system (1) and the adjoint system (28). We start by solving the state equations with a guess for the controls over the simulated time using the fourth-order Runge-Kutta iterative scheme method. The adjoint equations are solved by the backward fourth-order Runge-Kutta scheme using the current iterations solutions of the state equations because of the transversality conditions (31). Furthermore, the controls are updated by using a convex combination of the previous controls and the value from the characterizations (30). This process is repeated and the iterations are stopped if the values of the unknowns at the previous iterations are very close to the ones at the present iteration [47]. Based on the fact that brucellosis is endemic in most of the sub-Saharan Africa countries and that one control cannot stop the disease transmission, we investigate the impacts of combining at least three control strategies in a period of six years. Moreover, the computation of real weights of the objective function is very involving and needs a lot of information. In view of the aforesaid, the weights of the objective function are theoretically chosen to be $A_{1}=15, A_{2}=20, A_{3}=5$, $A_{4}=10, B_{1}=15, B_{2}=10, B_{3}=10$, and $B_{4}=10$ just to concede the control strategies proposed in this paper, and the parameter values used are in Table 3 . The initial state variables are chosen as $S_{c}(0)=200, I_{c}(0)=10, S$ ${ }_{s}(0)=200, I_{s}(0)=5, S_{h}(0)=50, I_{h}(0)=10$, $R_{h}(0)=5$, and $B(0)=100$.

The parameter values used in our computations are mainly from [3], a literature similar to this work.

8.1. Strategy A: Optimal Vaccination, Gradual Culling, and Environmental Sanitation. Under this strategy, the effectiveness of vaccination of ruminant control $u_{1}$, gradual culling of seropositive ruminants $u_{2}$, and environmental hygiene and sanitation $u_{3}$ are used to minimize the objective function $J$, whereas personal protection in humans control $u_{4}$ is set to zero. Figure 4 illustrates the trends of the infective classes.

Figure 4 shows that combination of the three control strategies leads to eradication of brucellosis from cattle in four years, small ruminants in two years, and humans in between two to three years. In case of no controls, the infective populations grow exponentially. 


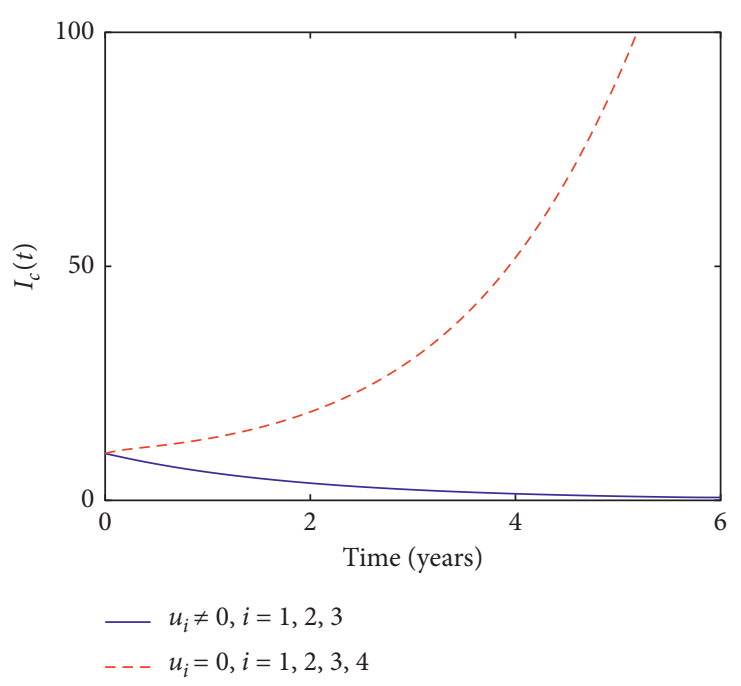

(a)

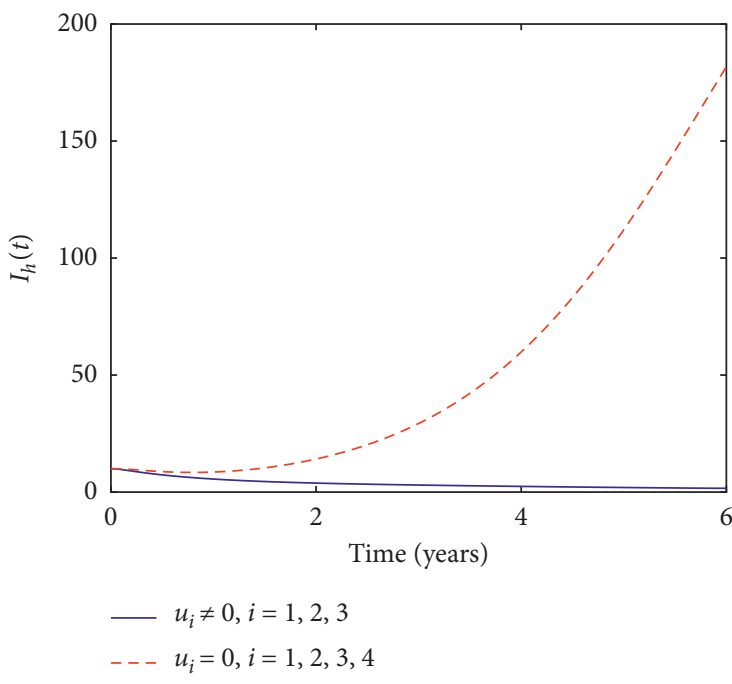

(c)

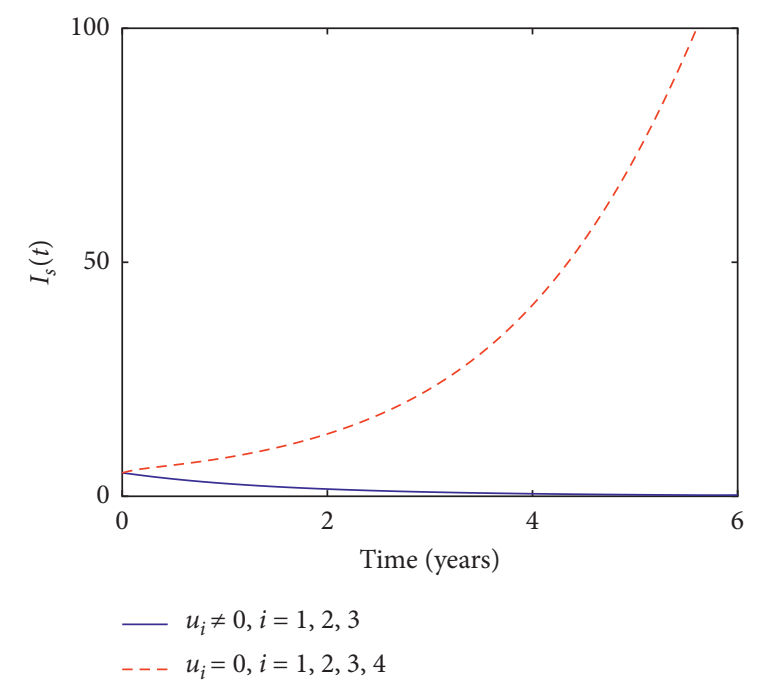

(b)

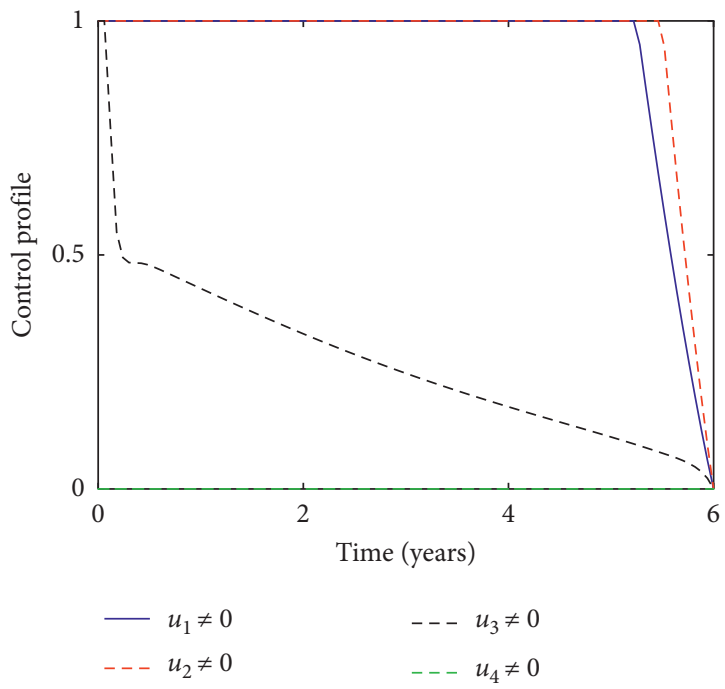

(d)

Figure 4: Dynamics of brucellosis with optimal vaccination, gradual culling of seropostive ruminants, and environmental hygiene and sanitation.

8.2. Strategy B: Optimal Vaccination, Gradual Culling, and Personal Protection. In this strategy, ruminant vaccination $u_{1}$, gradual culling of seropositive ruminants $u_{2}$, and personal protection in humans $u_{4}$ are used to optimize the objective function $J$ while the environmental hygiene sanitation control $u_{3}$ is set to zero. Figure 5 illustrates the variations in the infective classes.

Figure 5 shows that the cattle population attains its disease equilibrium in less than four years, whereas the disease can be eradicated from the small ruminants and humans in less than two years if the combination of the three controls is applied. In case of no control, the infected populations grow exponentially.

8.3. Strategy C: Optimal Vaccination, Environmental Hygiene, and Personal Protection. In this strategy, ruminant vaccination control $u_{1}$, environmental sanitation $u_{3}$, and personal protection $u_{4}$ are used to optimize the objective function $J$ while gradual culling of seropostive ruminants control $u_{2}$ is set to zero. Figure 6 illustrates the variations in the seropositive populations.

It can be seen from Figure 6 that the combination of the three control strategies leads to elimination of the infective humans and consequently a disease-free equilibrium is attained within a two years period. Furthermore, the small ruminant population attains its disease-free equilibrium point in five years and that of cattle will attain its equilibrium point in more than six years. In case of no controls, the number of infective individuals increases.

8.4. Strategy D: Optimal Gradual Culling, Environmental Hygiene, and Personal Protection. In this strategy, gradual culling of seropostive ruminants $u_{2}$, environmental hygiene and sanitation $u_{3}$, and personal protection $u_{4}$ are used to 


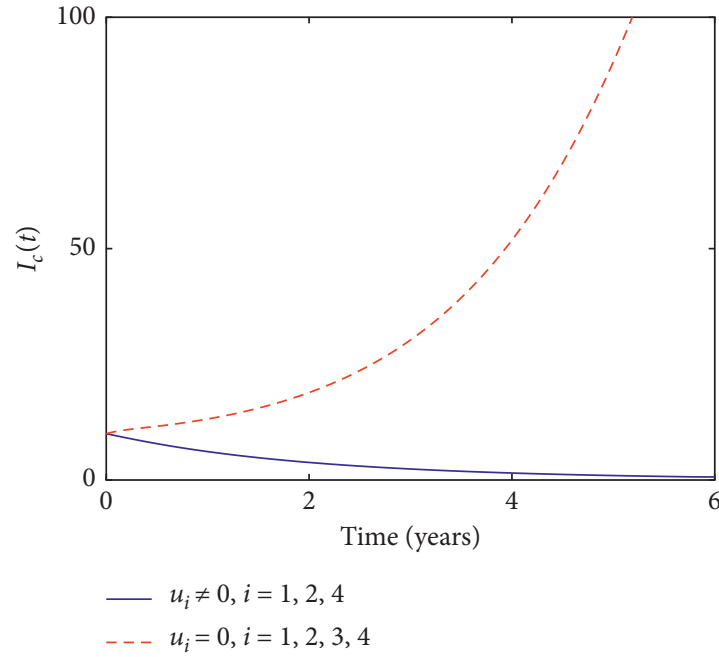

(a)

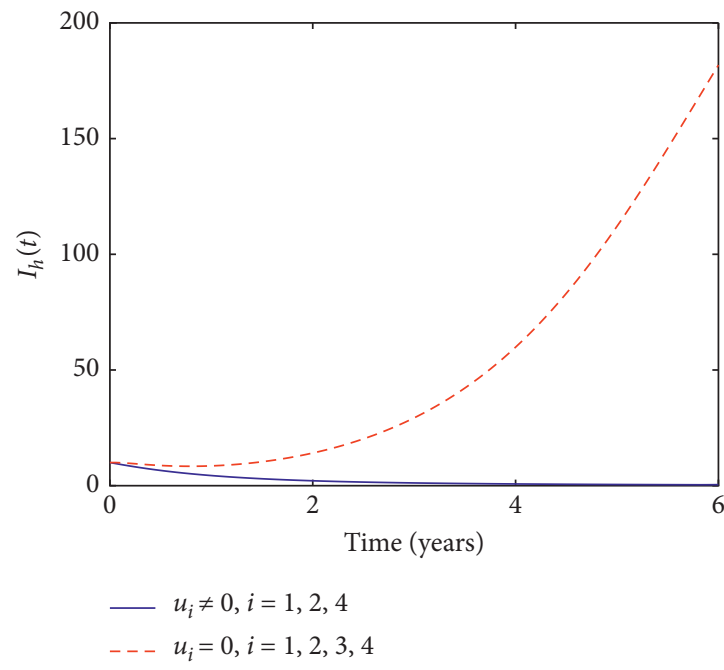

(c)

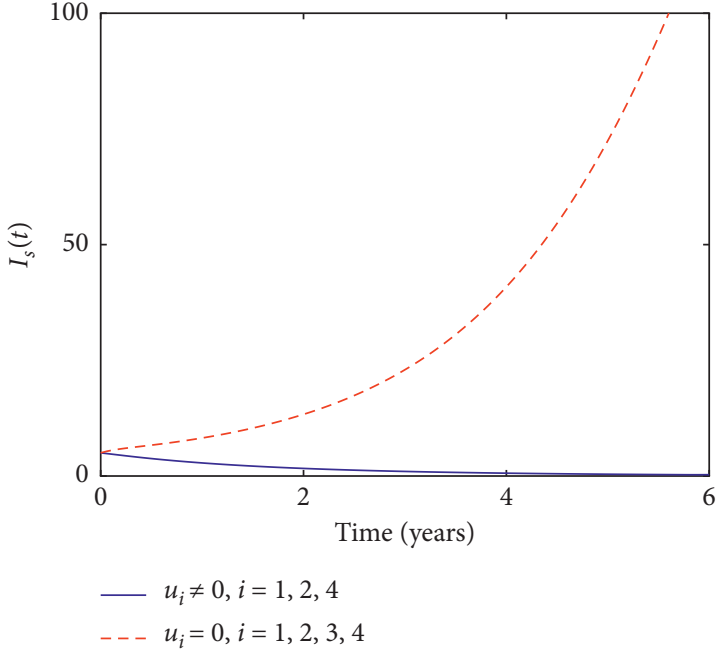

(b)

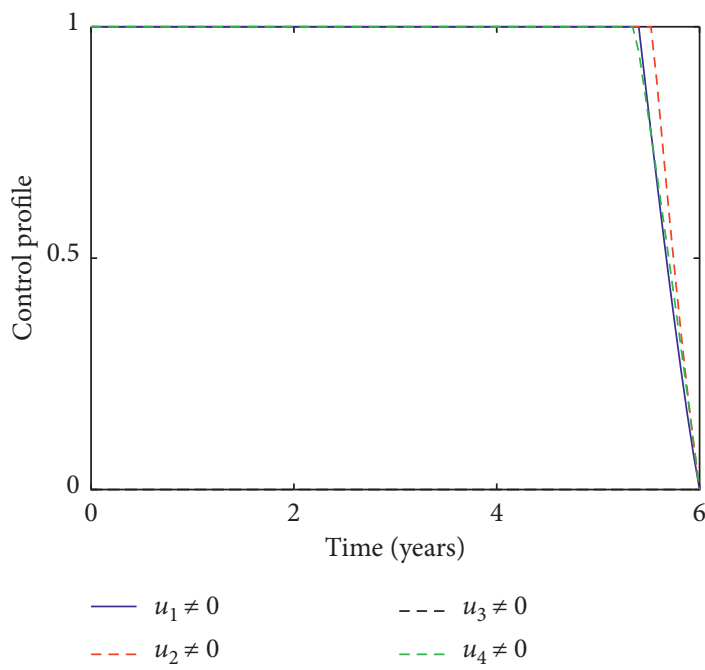

(d)

FIGURE 5: Dynamics of brucellosis with optimal vaccination, gradual culling of seropositive ruminants, and personal protection controls.

minimize the objective function $J$, whereas ruminants vaccination $u_{1}$ is set to zero. Figure 7 illustrates the variations in the seropositive populations.

It can be seen from Figure 7 that optimal implementation of gradual culling of seropostive ruminants, environmental hygiene, and personal protection reduces the number of infected human to zero in a period of less than two years, whereas the infected ruminants does not go to zero in a period of more than six years. This implies that implementation the three interventions under consideration does not make the ruminants population attain their diseasefree equilibrium points. Thus, this strategy is not mathematically recommended.

8.5. Strategy E: Optimal Vaccination, Gradual Culling, Environmental Sanitation, and Personal Protection. With this strategy, the combination of the four control strategies under consideration, ruminants vaccination, $u_{1}$, gradual culling of seropositive ruminants, $u_{2}$, environmental hygiene and sanitation, $u_{3}$, and personal protection, $u_{4}$, are used to optimize the objective function $J$. Figure 8 illustrates the variations in the infective populations.

Figure 8 shows that due to the combination of the four control strategies, the number of infected small ruminants and humans decreases to zero in less than two years while that of infective cattle reduces to zero in less than four years. In case of no controls, the number of infective populations grows exponentially.

\section{Cost-Effectiveness Analysis}

In this section, the cost effectiveness of the control strategies $\mathrm{A}, \mathrm{B}, \mathrm{C}, \mathrm{D}$, and $\mathrm{E}$ is analyzed using the Incremental CostEffectiveness Ratio (ICER), as described in [48-51]. The choice of the method is based on the fact that it allows the comparison between the cost effectiveness of combination of at least two of the controls. In particular, we compare two 


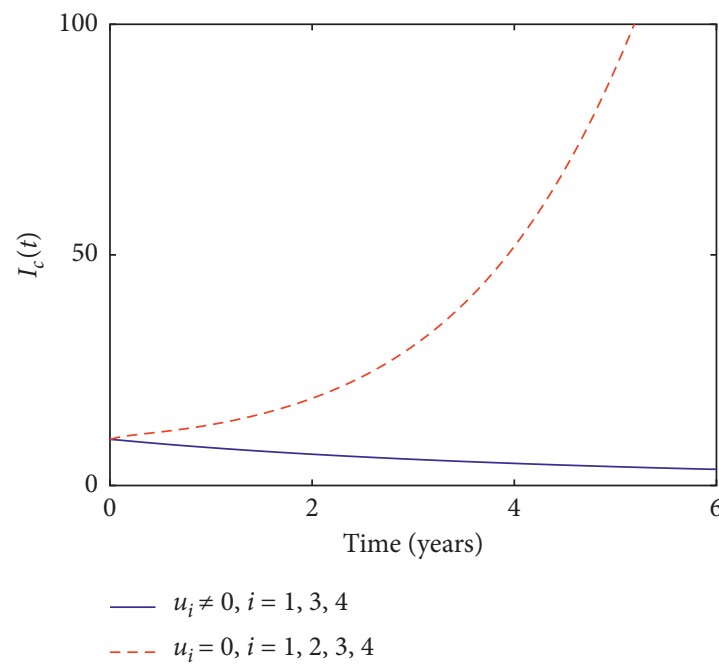

(a)

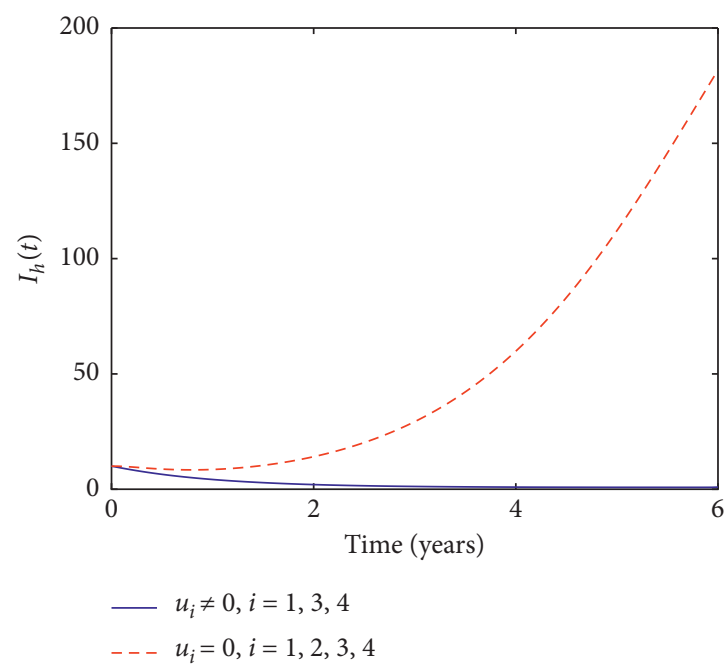

(c)

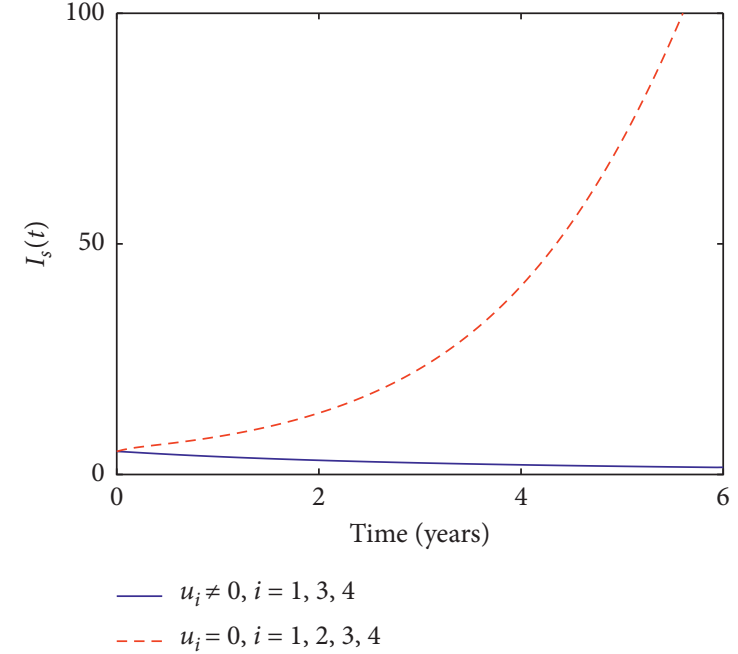

(b)

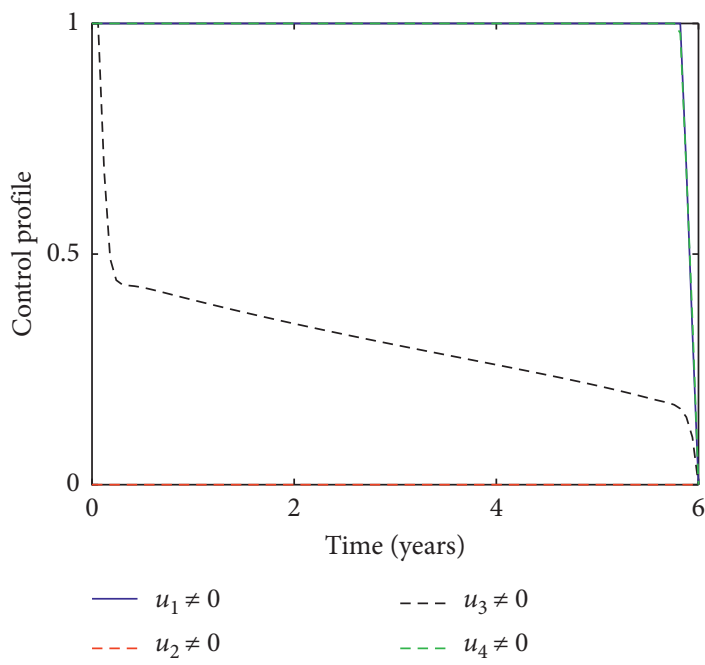

(d)

FIGURE 6: Dynamics of brucellosis with optimal vaccination, environmental hygiene, and personal protection controls.

competing intervention strategies incrementally; one intervention is being compared with the next less-effective alternative. ICER is defined by the difference in cost between two possible interventions divided by the difference in their outcome, given that they compete for the same resource. Mathematically,

$$
\text { ICER for } X=\frac{\text { Cost of intervention } X-\text { Cost of intervention } Y}{\text { Effect of intervention } X-\text { Effect of intervention } Y}
$$

where $X$ and $Y$ are the two intervention strategies being compared in this case, and the effect or benefits in health status are measured in terms of quality-adjusted life years (QALYs) gained or lost. In this approach, alternatives that are more expensive and less effective are excluded. Following the numerical simulation results of model (1), we rank the five strategies in order of increasing effectiveness measured as total infections averted and compute the ICER for every two competing strategies, and the results are presented in Table 4.
From Table 4, we see that strategy D is the most expensive and less effective strategy; thus, we exclude it from the set of alternatives so it does not consume limited resources. The recalculated ICER for remaining four alternatives is in Table 5 .

Table 5 shows that strategy $\mathrm{C}$ is the most expensive and less effective strategy; thus, we exclude it from the set of alternatives so it does not consume limited resources. Table 6 presents the recalculated ICER for remaining three alternatives. 


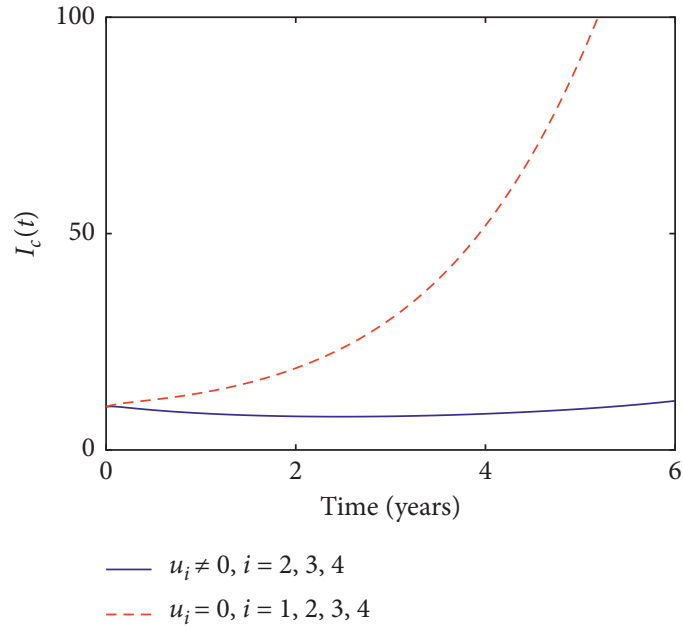

(a)

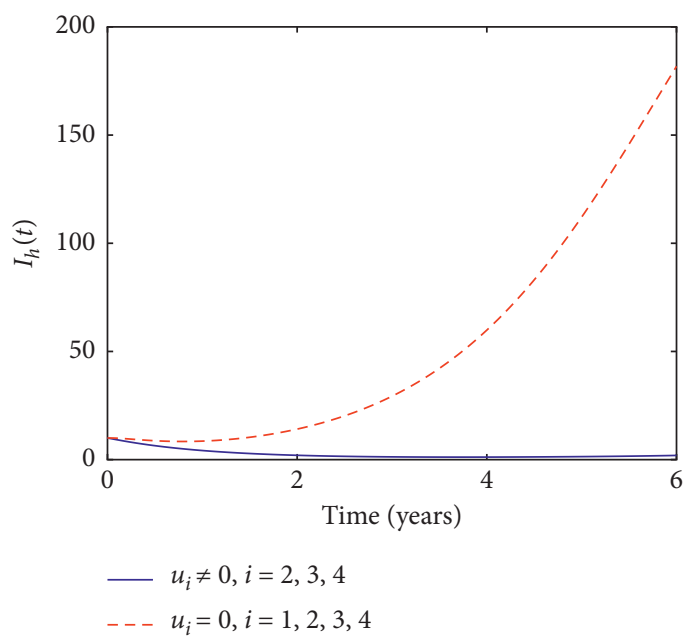

(c)

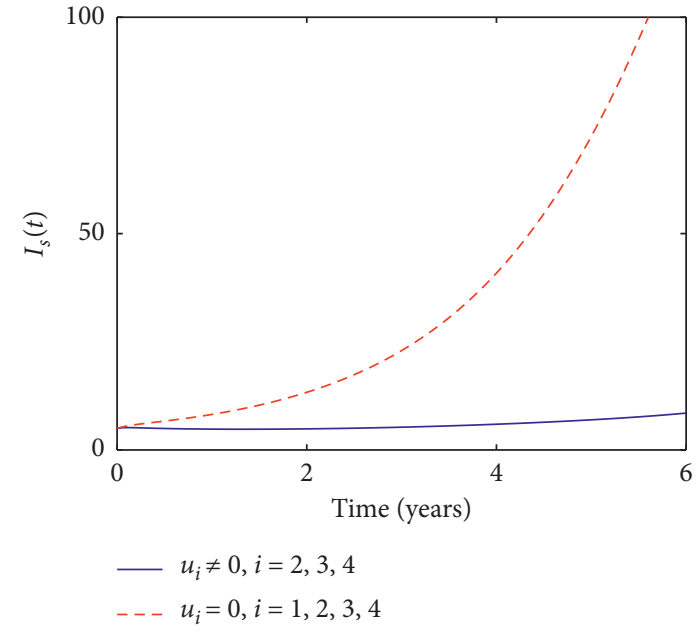

(b)

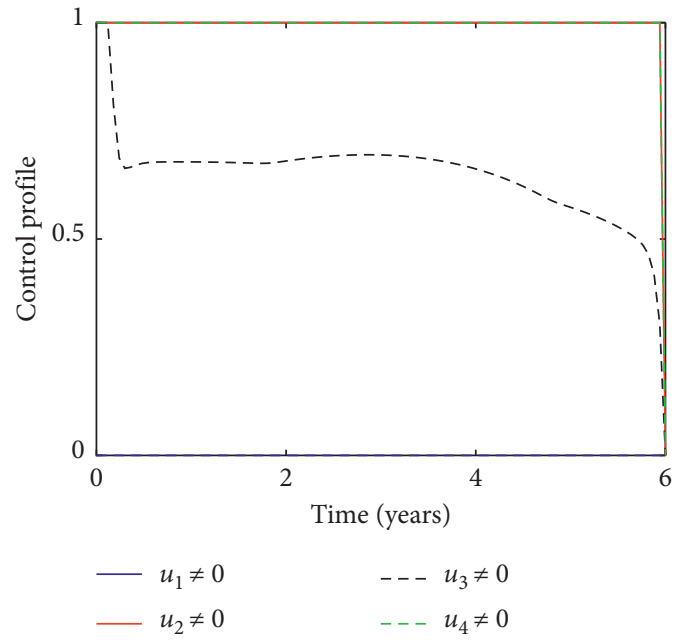

(d)

FIGURE 7: Dynamics of brucellosis with optimal gradual culling of seropostive ruminants, environmental sanitation, and personal protection controls.

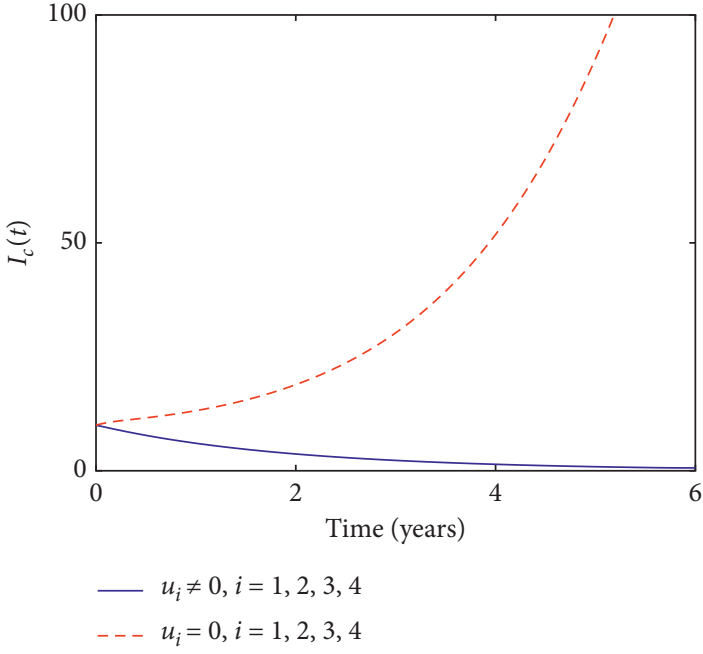

(a)

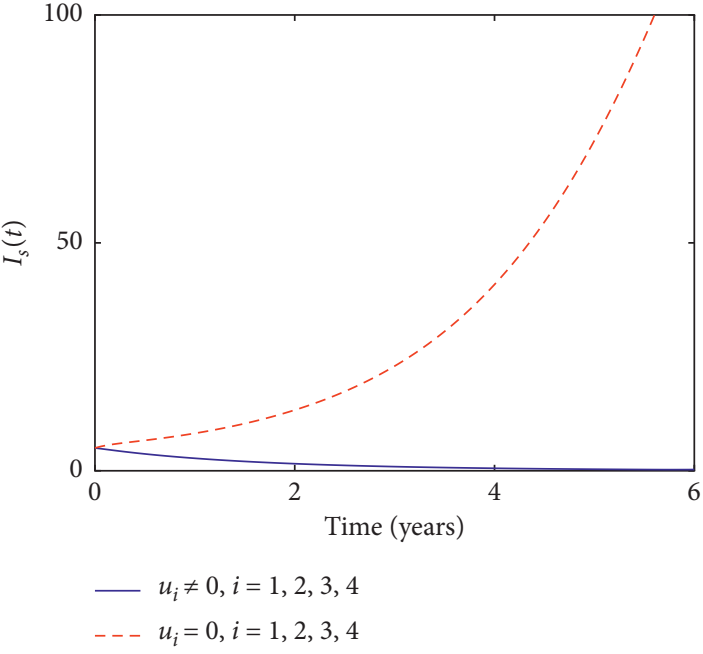

(b)

Figure 8: Continued. 


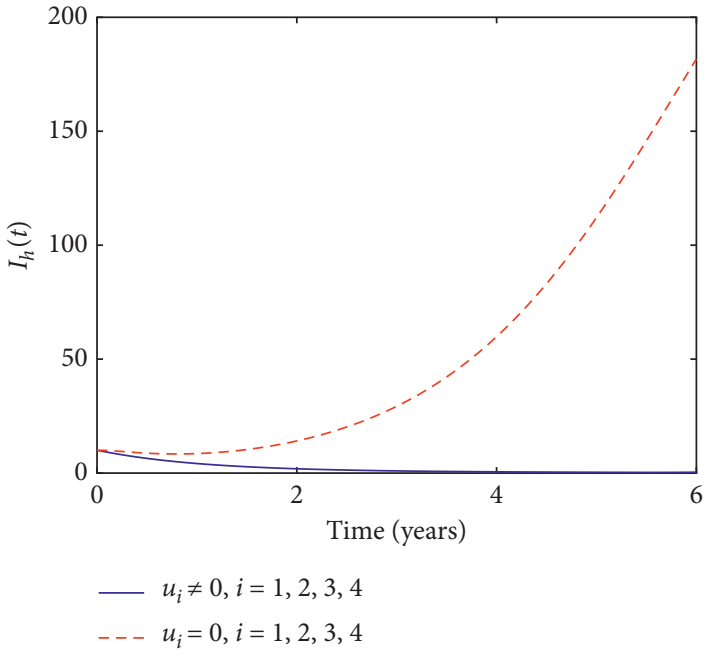

(c)

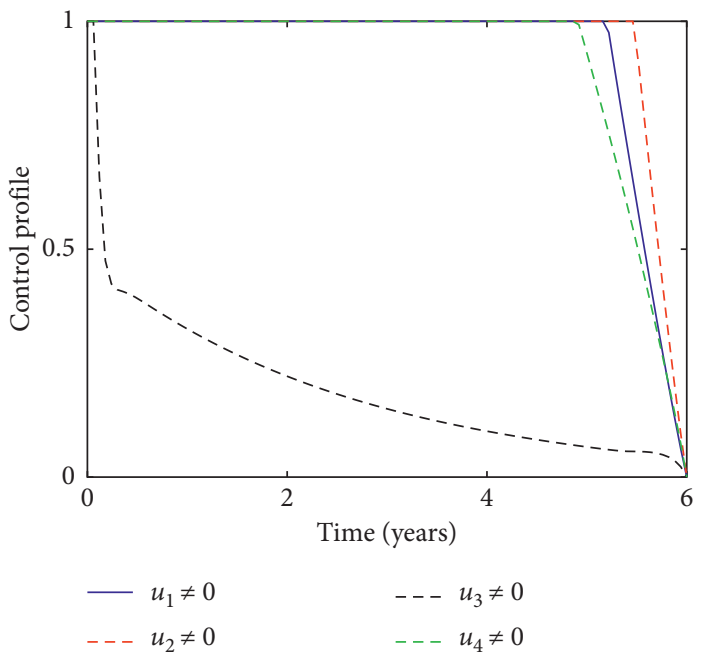

(d)

FiguRE 8: Dynamics of brucellosis with optimal vaccination, gradual culling of seropositive ruminants, environmental sanitation, and personal protection controls.

TABLE 4: ICER for alternative control strategies A, B, C, D, and E.

\begin{tabular}{lccc}
\hline Strategy & Total infection averted & Total cost $(\$)$ & ICER \\
\hline Strategy A & 12953 & 4795.7 & 0.3702 \\
Strategy B & 19789 & 4368 & -0.0625 \\
Strategy D & 20346 & 45535 & 73.9075 \\
Strategy C & 21409 & 13169 & -30.4478 \\
Strategy E & 24832 & 4047 & -2.6649 \\
\hline
\end{tabular}

TABLE 5: ICER for alternative control strategies A, B, C, and E.

\begin{tabular}{lccc}
\hline Strategy & Total infection averted & Total cost $(\$)$ & ICER \\
\hline Strategy A & 12953 & 4795.7 & 0.3702 \\
Strategy B & 19789 & 4368 & -0.0625 \\
Strategy C & 21409 & 13169 & 5.4324 \\
Strategy E & 24832 & 4047 & -2.6649 \\
\hline
\end{tabular}

TABLE 6: ICER for alternative control strategies A, B, and E.

\begin{tabular}{lccc}
\hline Strategy & Total infection averted & Total cost $(\$)$ & ICER \\
\hline Strategy A & 12953 & 4795.7 & 0.3702 \\
Strategy B & 19789 & 4368 & -0.0625 \\
Strategy E & 24832 & 4047 & -0.0638 \\
\hline
\end{tabular}

The comparison between strategies A and B shows a cost saving of $\$ 0.0625$ for strategy B over strategy A. The lower ICER for strategy B indicates that strategy A is strongly dominated; that is, strategy A is more costly and less effective than strategy B. Therefore, we exclude strategy A from the set of alternatives so it does not consume limited resources. The recalculated ICER is shown in Table 7.

Moreover, Table 7 shows a cost saving of $\$ 0.0638$ for strategy E over strategy B. In this case, the incremental cost is
TABLE 7: ICER for alternative control strategies B and E.

\begin{tabular}{lccc}
\hline Strategy & Total infection averted & Total cost $(\$)$ & ICER \\
\hline Strategy B & 19789 & 4368 & 0.2208 \\
Strategy E & 24832 & 4047 & -0.0638 \\
\hline
\end{tabular}

negative and the incremental effect is positive (south-east quadrant of the cost-effectiveness plane), and the intervention is unequivocally cost effective (it is dominant, achieving better outcomes at lower cost) [48-50]. The lower ICER for strategy $\mathrm{E}$ indicates that strategy $\mathrm{B}$ is more costly and less effective than strategy E; in other words, strategy $\mathrm{E}$ is more effective and saves money compared with the strategy B. Hence, strategy B is excluded from the set of alternatives so it does not consume limited resources. Thus, strategy $\mathrm{E}$ which is the combination of vaccination, gradual culling through slaughter of seropositive cattle and small ruminants, environmental sanitation, and personal protection in humans has the least ICER and therefore is more cost-effective strategy than all alternatives under consideration.

\section{Conclusion}

This paper aimed at formulating and analyzing a mathematical model for the impacts of different control options to the transmission dynamics of Brucellosis. We focused on livestock vaccination; gradual culling through slaughter of seropositive cattle and small ruminants; environmental hygiene and sanitation; and personal protection in humans. Pontryagin's Maximum Principle approach and incremental cost-effectiveness ratio were, respectively, used to analyze the optimal control problem and the cost effectiveness of the control strategies. Findings in both optimal control and costeffectiveness analysis revealed that combination of vaccination, gradual culling of seropositive cattle and small ruminants, environmental hygiene, and personal protection in 
humans is unequivocally best control strategy as it has high impact with lower cost of controlling the disease. It was revealed further that strategy $B$ which is the combination of vaccination, gradual culling through slaughter of seropositive cattle and small ruminants, and personal protection in humans is the second cost-effective strategy, followed by strategy A which is the combination of vaccination, gradual culling of seropositive cattle and small ruminants, and environmental hygiene. Furthermore, strategy $\mathrm{C}$ which is the combination of vaccination of livestock, environmental hygiene, and personal protection in humans is less effective in controlling the disease while strategy $\mathrm{D}$ is the least among the alternatives and cannot be recommended for implementation as it has lower impact and higher cost. Critical analysis of the four control options showed that vaccination of susceptible livestock and gradual culling of seropostive cattle and small ruminants are the key parameter for any design of a control option. This paper recommends that, for effective and efficiency brucellosis transmission control, the combination of vaccination, gradual culling of seropositive cattle and small ruminants, environmental hygiene, and personal protection in humans should be adopted.

\section{Data Availability}

The data supporting the findings in the article were derived as follows. We used the set of parameter values mainly from Li et al. [3], an article similar to this work, while unavailable data, especially values of parameters, are estimated for the purpose of verifying results of the mathematical analyses of the model developed in the manuscript.

\section{Conflicts of Interest}

The authors declare no conflicts of interest regarding the publication of this paper.

\section{Acknowledgments}

Authors acknowledge the contributions from One Health and Tanzania Veterinary Association (TVA) for an opportunity to make a presentation in the "First One Health Conference, embedding the 37th TVA Scientific Conference, held on November 27th-29th, 2019, at Arusha International Conference Centre, Arusha-Tanzania" for comments that led to improvement of this work.

\section{References}

[1] WHO, "Brucellosis in humans and animals," 2018, http:// www.who.int/csr/resources/publications/Brucellosis.pdf.

[2] F. P. Poester, L. E. Samartino, and R. L. Santos, "Pathogenesis and pathobiology of brucellosis in livestock," Revue Scientifique et Technique de l'OIE, vol. 32, no. 1, pp. 105-115, 2013.

[3] M.-T. Li, G.-Q. Sun, Y.-F. Wu, J. Zhang, and Z. Jin, "Transmission dynamics of a multi-group brucellosis model with mixed cross infection in public farm," Applied Mathematics and Computation, vol. 237, pp. 582-594, 2014.

[4] CFSPH, "Brucellosis brucella abortus," 2018, http://cfsph. iastate.edu/Factsheets/pdfs/brucellosis_abortus.pdf.
[5] E. Schelling, C. Diguimbaye, S. Daoud et al., "Brucellosis and Q-fever seroprevalences of nomadic pastoralists and their livestock in Chad," Preventive Veterinary Medicine, vol. 61, no. 4, pp. 279-293, 2003.

[6] R. A. Greenfield, D. A. Drevets, L. J. Machado, G. W. Voskuhl, P. Cornea, and M. S. Bronze, "Bacterial pathogens as biological weapons and agents of bioterrorism," The American Journal of the Medical Sciences, vol. 323, no. 6, pp. 299-315, 2002.

[7] J. Zhang, G. Sun, X. Sun et al., "Prediction and control of brucellosis transmission of dairy cattle in Zhejiang province, China," PLoS One, vol. 9, no. 11, Article ID e108592, 2014.

[8] F. Zhang, Z. Li, X. La et al., "Multiple-locus variable-number tandem-repeat analysis of Brucella isolates from patients in Xinjiang, China," International Journal of Clinical and Experimental Medicine, vol. 8, no. 9, pp. 15716-15723, 2015.

[9] B. E. Aïnseba, C. Benosman, and P. Magal, "A model for ovine brucellosis incorporating direct and indirect transmission," Journal of Biological Dynamics, vol. 4, no. 1, pp. 2-11, 2010.

[10] J. Zinsstag, F. Roth, D. Orkhon et al., "A model of animalhuman brucellosis transmission in Mongolia," Preventive Veterinary Medicine, vol. 69, no. 1-2, pp. 77-95, 2005.

[11] M. N. Xavier, T. A. Paixao, A. B. den Hartigh, R. M. Tsolis, and R. L. Santos, "Pathogenesis of Brucella spp." The Open Veterinary Science Journal, vol. 4, no. 1, pp. 109-118, 2010.

[12] Medscape, "Brucellosis pathogenicity," 2018, https:// emedicine.medscape.com/article/213430-overview.

[13] K. A. Franc, R. C. Krecek, B. N. Häsler, and A. M. ArenasGamboa, "Brucellosis remains a neglected disease in the developing world," BMC Public Health, vol. 18, no. 1, pp. 18-125, 2018.

[14] M. Ducrotoy, W. J. Bertu, G. Matope et al., "Brucellosis in Sub-Saharan Africa: current challenges for management, diagnosis and control," Acta Tropica, vol. 165, pp. 179-193, 2017.

[15] M. Yilma, G. Mamo, and B. Mammo, "Review on brucellosis sero-prevalence and ecology in livestock and human population of Ethiopia," Achievements in the Life Sciences, vol. 10, no. 1, pp. 80-86, 2016.

[16] A. S. Dean, L. Crump, H. Greter, E. Schelling, and J. Zinsstag, "Global burden of human brucellosis: a systematic review of disease frequency," PLoS Neglected Tropical Diseases, vol. 6, no. 10, pp. 1-9, 2012.

[17] CDC, Brucellosis Signs and Symptoms, CDC, Atlanta, GA, USA, 2018, https://www.cdc.gov/brucellosis/symptoms/ index.html.

[18] K. John, J. Fitzpatrick, N. French et al., "Quantifying risk factors for human brucellosis in rural northern Tanzania," PLoS One, vol. 5, no. 4, Article ID e9968, 2010.

[19] G. Pappas, P. Papadimitriou, N. Akritidis, L. Christou, and E. V. Tsianos, "The new global map of human brucellosis," The Lancet Infectious Diseases, vol. 6, no. 2, pp. 91-99, 2006.

[20] G. Tumwine, E. Matovu, J. D. Kabasa, D. O. Owiny, and S. Majalija, "Human brucellosis: sero-prevalence and associated risk factors in agro-pastoral communities of Kiboga district, Central Uganda," BMC Public Health, vol. 15, no. 1, pp. 1-8, 2015.

[21] J. Kitaly, "Bovine brucellosis in Government parastatal and Ujamaa village dairy farms in central zone of Tanzania: assessment of Control measures in some farms," in Proceedings of the 2nd Tanzania Veterinary Association Scientific Conference, pp. 24-30, Arusha, Tanzania, December 1984.

[22] E. S. Swai and L. Schoonman, "Human brucellosis: seroprevalence and risk factors related to high risk occupational 
groups in Tanga municipality, Tanzania," Zoonoses and Public Health, vol. 56, no. 4, pp. 183-187, 2009.

[23] M. Carugati, H. M. Biggs, M. J. Maze et al., "Incidence of human brucellosis in the Kilimanjaro region of Tanzania in the periods 2007-2008 and 2012-2014," Transactions of The Royal Society of Tropical Medicine and Hygiene, vol. 112, no. 3, pp. 136-143, 2018.

[24] G. M. Shirima, The epidemiology of brucellosis in animals and humans in Arusha and Manyara regions in Tanzania, Ph.D. thesis, University of Glasgow, Glasgow, Scotland, 2005.

[25] E. Swai and L. Schoonman, "A survey of zoonotic diseases in trade cattle slaughtered at Tanga city abattoir: a cause of public health concern," Asian Pacific Journal of Tropical Biomedicine, vol. 2, no. 1, pp. 55-60, 2012.

[26] A. G. Alhamada, I. Habib, A. Barnes, and I. Robertson, "Risk factors associated with brucella seropositivity in sheep and goats in Duhok province, Iraq," Veterinary Sciences, vol. 4, no. 65, pp. 1-9, 2017.

[27] N. Nyerere, L. S. Luboobi, S. C. Mpeshe, and G. M. Shirima, "Mathematical model for the infectiology of brucellosis with some control strategies," New Trends in Mathematical Sciences, vol. 7, no. 4, pp. 387-405, 2019.

[28] N. Nyerere, L. S. Luboobi, S. C. Mpeshe, and G. M. Shirima, "Mathematical model for brucellosis transmission dynamics in livestock and human populations," Communications in Mathematical Biology and Neuroscience, vol. 2020, no. 3, pp. 1-29, 2020.

[29] Q. Hou, X. Sun, J. Zhang, Y. Liu, Y. Wang, and Z. Jin, "Modeling the transmission dynamics of sheep brucellosis in inner Mongolia autonomous region, China," Mathematical Biosciences, vol. 242, no. 1, pp. 51-58, 2013.

[30] C. Li, Z.-G. Guo, and Z.-Y. Zhang, "Transmission dynamics of a brucellosis model: basic reproduction number and global analysis," Chaos, Solitons \& Fractals, vol. 104, pp. 161-172, 2017.

[31] B. Nannyonga, G. G. Mwanga, and L. S. Luboobi, "An optimal control problem for ovine brucellosis with culling," Journal of Biological Dynamics, vol. 9, no. 1, pp. 198-214, 2015.

[32] P. O. Lolika, C. Modnak, and S. Mushayabasa, "On the dynamics of brucellosis infection in bison population with vertical transmission and culling," Mathematical Biosciences, vol. 305, pp. 42-54, 2018.

[33] F. Roth, J. Zinsstag, D. Orkhon et al., "Human health benefits from livestock vaccination for brucellosis: case study," Bulletin of the World Health Organization, vol. 81, pp. 867-876, 2003.

[34] O. Diekmann, J. A. P. Heesterbeek, and J. A. J. Metz, "On the definition and the computation of the basic reproduction ratio $R_{0}$ in models for infectious diseases in heterogeneous populations," Journal of Mathematical Biology, vol. 28, no. 4, pp. 365-382, 1990.

[35] O. Diekmann, J. A. P. Heesterbeek, and M. G. Roberts, “The construction of next-generation matrices for compartmental epidemic models," Journal of the Royal Society Interface, vol. 7, no. 47, pp. 873-885, 2010.

[36] D. Okuonghae and A. Korobeinikov, "Dynamics of tuberculosis: the effect of direct observation therapy strategy (DOTS) in Nigeria," Mathematical Modelling of Natural Phenomena, vol. 2, no. 1, pp. 113-128, 2007.

[37] A. Palanduz, S. Palanduz, K. Guler, and N. Guler, "Brucellosis in a mother and her young infant: probable transmission by breast milk," International Journal of Infectious Diseases, vol. 4, no. 1, pp. 1-55, 2000.

[38] M. J. Michael, Brucellosis in Humans and Animals, World Health Organization, Geneva, Switzerland, 2006.

[39] O. Mesner, K. Riesenberg, N. Biliar et al., "The many faces of human-to-human transmission of brucellosis: congenital infection and outbreak of nosocomial disease related to an unrecognized clinical case," Clinical Infectious Diseases, vol. 45, no. 12, pp. e135-e140, 2007.

[40] E. Meltzer, Y. Sidi, G. Smolen, M. Banai, S. Bardenstein, and E. Schwartz, "Sexually transmitted brucellosis in humans," Clinical Infectious Diseases, vol. 51, no. 2, pp. e12-e15, 2010.

[41] A. El-Sayed and W. Awad, "Brucellosis: evolution and expected comeback," International Journal of Veterinary Science and Medicine, vol. 6, no. 1, pp. s31-s35, 2018.

[42] F. F. Tuon, R. B. Gondolfo, and N. Cerchiari, "Human-tohuman transmission of Brucella-a systematic review," Tropical Medicine \& International Health, vol. 22, no. 5, pp. 539-546, 2017.

[43] M. Viana, G. M. Shirima, K. S. John et al., "Integrating serological and genetic data to quantify cross-species transmission: brucellosis as a case study," Parasitology, vol. 143, no. 7, pp. 821-834, 2016.

[44] D. L. Lukes, Differential Equations: Classical to Controlled, Vol. 162, Academic Press, vol.162, New York, NY, USA, 1982.

[45] L. S. Pontryagin, V. G. Boltyanskii, R. V. Gamkrelidze, and E. F. Mishchenko, The Mathematical Theory of Optimal Processes, Routledge, Abingdon, UK, 1962.

[46] W. H. Fleming and R. W. Rishel, Deterministic and Stochastic Optimal Control, vol. 1, Springer Science \& Business Media, Berlin, Germany, 2012.

[47] S. Lenhart and J. T. Workman, Optimal Control Applied to Biological Models, CRC Press, London, UK, 2007.

[48] D. Hartwell, J. Jones, L. Baxter, and J. Shepherd, "Peginterferon alfa and ribavirin for chronic hepatitis $C$ in patients eligible for shortened treatment, re-treatment or in HCV/HIV co-infection: a systematic review and economic evaluation," Health Technology Assessment, vol. 15, no. 17, pp. 1-210, 2011.

[49] R. M. Klok and M. J. Postma, "Four quadrants of the costeffectiveness plane: some considerations on the south-west quadrant," Expert Review of Pharmacoeconomics \& Outcomes Research, vol. 4, no. 6, pp. 599-601, 2004.

[50] P. A. McFarlane and A. M. Bayoumi, "Acceptance and rejection: cost-effectiveness and the working nephrologist," Kidney International, vol. 66, no. 5, pp. 1735-1741, 2004.

[51] K. O. Okosun, O. Rachid, and N. Marcus, "Optimal control strategies and cost-effectiveness analysis of a malaria model," BioSystems, vol. 111, no. 2, pp. 83-101, 2013. 